\title{
Perceptually Lossless Compression for Mastcam Multispectral Images: A Comparative Study
}

\author{
Chiman Kwan*, Jude Larkin \\ Applied Research LLC, Rockville, MD, USA \\ Email:*chiman.kwan@arllc.net
}

How to cite this paper: Kwan, C. and Larkin, J. (2019) Perceptually Lossless Compression for Mastcam Multispectral Images: A Comparative Study. Journal of Signal and Information Processing, 10, 139-166. https://doi.org/10.4236/jsip.2019.104009

Received: October 13, 2019

Accepted: November 25, 2019

Published: November 28, 2019

Copyright (อ 2019 by author(s) and Scientific Research Publishing Inc. This work is licensed under the Creative Commons Attribution International License (CC BY 4.0).

http://creativecommons.org/licenses/by/4.0/

\section{(c) (i) Open Access}

\begin{abstract}
The two mast cameras, Mastcams, onboard Mars rover Curiosity are multispectral imagers with nine bands in each. Currently, the images are compressed losslessly using JPEG, which can achieve only two to three times of compression. We present a comparative study of four approaches to compressing multispectral Mastcam images. The first approach is to divide the nine bands into three groups with each group having three bands. Since the multispectral bands have strong correlation, we treat the three groups of images as video frames. We call this approach the Video approach. The second approach is to compress each group separately and we call it the split band (SB) approach. The third one is to apply a two-step approach in which the first step uses principal component analysis (PCA) to compress a nine-band image cube to six bands and a second step compresses the six PCA bands using conventional codecs. The fourth one is to apply PCA only. In addition, we also present subjective and objective assessment results for compressing RGB images because RGB images have been used for stereo and disparity map generation. Five well-known compression codecs, including JPEG, JPEG2000 (J2K), X264, X265, and Daala in the literature, have been applied and compared in each approach. The performance of different algorithms was assessed using four well-known performance metrics. Two are conventional and another two are known to have good correlation with human perception. Extensive experiments using actual Mastcam images have been performed to demonstrate the various approaches. We observed that perceptually lossless compression can be achieved at 10:1 compression ratio. In particular, the performance gain of the SB approach with Daala is at least $5 \mathrm{dBs}$ in terms peak signal-to-noise ratio (PSNR) at 10:1 compression ratio over that of JPEG. Subjective comparisons also corroborated with the objective metrics in that perceptually lossless compression can be achieved even at 20 to 1 compression.
\end{abstract}




\section{Keywords}

Perceptually Lossless Compression, Mastcam Images, JPEG, J2K, X264, X265, Daala, Multispectral, PCA, Video Compression

\section{Introduction}

Image compression is a well-developed field [1]. People have focused on lossless compression for secured data storage/transmission, or aggressive lossy compression for mobile applications in the past. Lossless compression can only achieve 2 to 3 times of compression. Aggressive lossy compression usually aims for 20 or more times of compression. Recently, we have been focusing on achieving something in the middle. That is, we aim at achieving perceptually lossless compression with a compression ratio of 10 . Such a requirement is necessary for many commercial and military applications where users want to achieve a compromise between compression quality and bandwidth usage.

Mars rover Curiosity has many instruments onboard for Mars data collection and in-situ surface characterization [2]. Alpha Particle X-ray Spectrometer (APXS) [3], Laser Induced Breakdown Spectrometer (LIBS) [4] [5], and Mastcam [6] [7] [8] [9] are well-known ones. Quite a few of these instruments are imagers that fight for limited bandwidth to transmit data back to Earth. Currently, the Mastcam images are all compressed using JPEG, a technology of 90's [10]. Although JPEG [10] is simple and efficient, the compression ratio for lossless compression can be at most between two to three times. There are new compression standards developed in the past two decades. Well-known video codecs include J2K [11], X264 [12], and X265 [13], which are also applicable to still image compression. J2K, X264, and X265 also provide lossless compression options. In some applications such as security monitoring where video quality is of prime importance, people are still using lossless image compression algorithms such as JPEG and J2K for compressing videos frame by frame.

JPEG, X264, and X265 are discrete cosine transform (DCT) based algorithms and $\mathrm{J} 2 \mathrm{~K}$ is wavelet based. About 15 years ago, there were some developments in DCT based algorithms where overlapped blocks known as lapped transforms (LT) were used to further improve the compression [14]. In the past few years, a group of researchers have incorporated LT [14] into an open source codec known as Daala [15]. Daala can be used for both still and video compression. There is also a lossless option.

In our earlier papers [16] [17], we have proposed and evaluated two approaches to Mastcam image compression. One of them is a two-step approach [16], which we first applied Principal Component Analysis (PCA) [18] [19] [20] [21] [22] to compress the nine-band Mastcam image cube to three bands or six bands and then a conventional codec (JPEG, J2K, X264, X265) was applied to further compress the three or six PCA bands. It was observed that using six PCA 
bands yielded better performance than that of using three bands. Another approach [17] is the split band (SB) approach, which splits the nine bands into three groups and apply still image compression to each group separately.

In this research, we present a thorough comparative of four approaches to Mastcam image compression. In addition to the two earlier approaches [16] [17], we propose a new video approach to compressing Mastcam images by treating the three groups of three bands as video frames. Moreover, we also include a study by using PCA only for compression. This is because PCA itself is a good compression technique. In all of the four approaches, we have added Daala in our experiments. That is, five image codecs in the literature (JPEG, J2K, X264, $\mathrm{X} 265$, and Daala) were evaluated to see which one can achieve perceptually lossless compression with compression ratio of 10:1. It is important to emphasize that perceptual performance assessment requires a suitable metric. Some conventional metrics such as peak signal-to-noise ratio (PSNR) and structural similarity (SSIM) may not match well with human's subjective evaluations. Recently, some researchers have developed metrics known as human visual system (HVS) and HVS with masking (HVSm) [23] that correlate well with human perceptions. We have incorporated HVS and HVSm into our comparative studies. Subjective evaluations for RGB Mastcam images have also been carried out. It was observed that perceptually lossless compression can be achieved even at 20 to 1 compression. Most importantly, at 10 to 1 compression, some objective metrics are 5 to $10 \mathrm{dBs}$ higher than JPEG when Daala was used.

The first key contribution of our project is to propose a video approach to compressing multispectral image data cube. This video approach can be applied to any multispectral or hyperspectral images. The second contribution is to compare the video approach with three alternatives (SB approach, two-step approach, and PCA only approach). The third contribution is a thorough comparison of the four approaches to Mastcam images. No one, except our group, has thoroughly studied this Mastcam image compression problem before.

Our paper is organized as follows. Section 2 summarizes the technical approach and its components. Section 3 summarizes all the experiments using actual images that are of interest to our customer. Finally, concluding remarks will be given in Section 4.

\section{Technical Approach}

\subsection{Proposed Compression Approaches for Mastcam Images}

\section{Video approach}

As mentioned earlier, we have four approaches to compression Mastcam images. Two approaches were described in earlier papers. In this research, we propose a video approach to compression. Our overall technical approach can be summarized as follows. First, some preprocessing steps are used to make sure the image height and width are even numbered, normalized, and in double. Second, the nine-band image cube is converted to three groups of three-band 
images. Third, since different codecs require different input formats, the images are saved to appropriate formats such as Y4m or YUV444. Fourth, we will apply the various compression algorithms (JPEG, J2K, X264, X265, and Daala) to the three-frame video and generate various performance metrics. There are four performance metrics. Each metric is computed by comparing the reconstructed nine-band cube with the original 9-band cube. The compression ratio is generated by comparing the size the compressed file with the size of the original image cube.

Figure 1 illustrates the work flow of the video approach. We include some details for some of the blocks.

- Pre-processing

The preprocessing has a few components. First, it is important to ensure the input image dimensions to have even numbers because some codecs may crash if the image size has odd dimensions. Second, the input image is normalized to double precision with values between 0 and 1 . Third, the different bands are saved into tiff format. Fourth, all the bands are written into YUV444 and Y4M formats.

- Video codecs

Different codecs have different requirements. For JPEG, we used FFMPEG to create a three-frame MPEG video with certain target quality parameter. The video is then decoded using FFMPEG. Finally, the decoded video is then converted to individual frames via the imread command in Matlab. For J2K, we used Matlab's video writer to create a J2K video with certain quality parameters. We then used Matlab's video reader to decode the video and the individual frames will be retrieved. For X264 and X265, the videos are encoded using the respective encoders with certain quality parameters. The video decoding was done within FFMPEG. For Daala, we directly used the Daala's functions for encoding and decoding.

- Performance evaluation

In the evaluation part, each frame is reconstructed and compared to the original input band. Four performance metrics have been used.

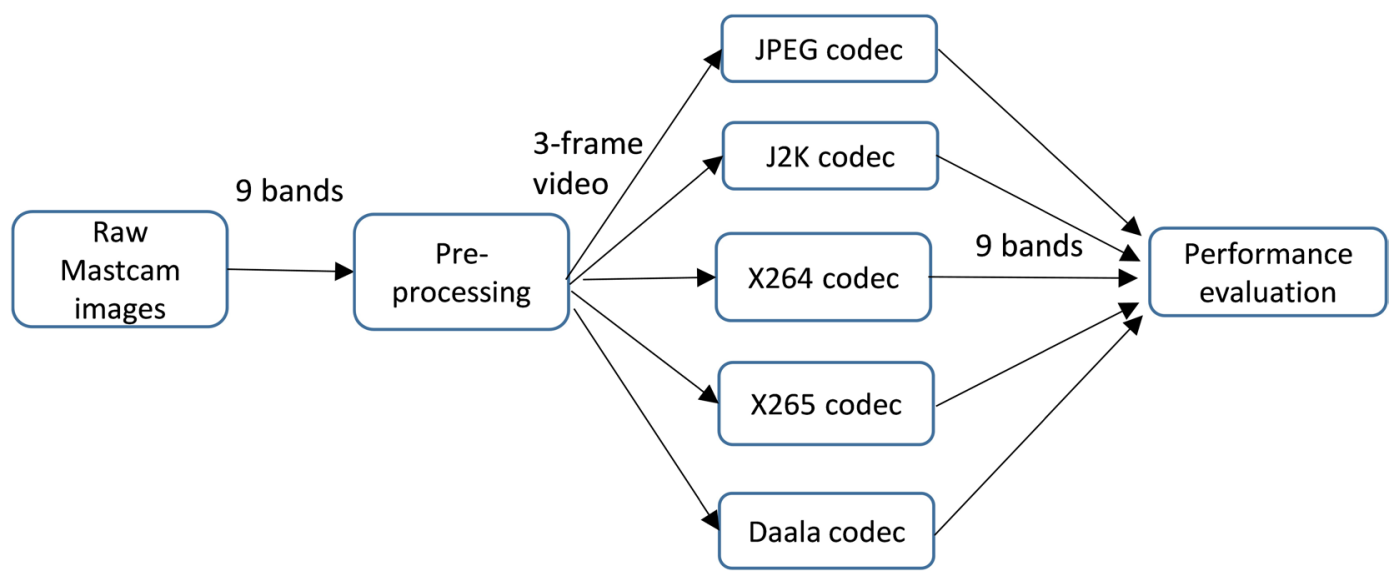

Figure 1. Proposed video approach to compressing Mastcam images. 


\section{Split Band (SB) Approach}

Here, we briefly summarize an alternative approach, which was presented in [17]. Details can be found in [17]. In [17], we did not include Daala. Here, we include new results with Daala as one of the codecs. The overall architecture is very similar to that of the video approach, except that each 3-band image is now compressed independently.

\section{Two-step approach}

Some preliminary results of this two-step approach were presented in [16]. The detailed signal flow can be found in Figure 2. The first step is to apply PCA to compress the nine-band Mastcam image cube to three or six bands. The second step is to compress the three or six bands using conventional codecs. In [16], we found that six-band has better performance. It should be noted that we did not include Daala in [16] because at that time, we did not know Daala has a newer version that outperforms an old version developed about 2 years ago.

\section{One-step PCA only approach}

It is well-known that PCA can be used for data compression. Actually, PCA, unlike DCT and wavelet, is a data dependent approach that yields optimal data compression. Figure 3 illustrates the PCA approach.

\section{Compressing RGB only}

The 5, 4, 2 bands in the Mastcam image cube are the RGB bands. We have performed a separate study specifically for RGB bands. It is similar to the SB approach.

\subsection{Brief Overview of Relevant Compression Algorithms}

In this paper, we will compare image codecs in the market and objectively evaluate different codecs and eventually recommend the best codec to our customer.

With the above in mind, we performed a brief summary of the existing high performance codecs.

\section{DCT based algorithms}

- JPEG [10]:

JPEG is the very first image compression standard. The video counterparts are the MPEG-1 and MPEG-2 standards.

- JPEG-XR [24]:

It was developed by Microsoft. The performance is comparable to JPEG-2000.

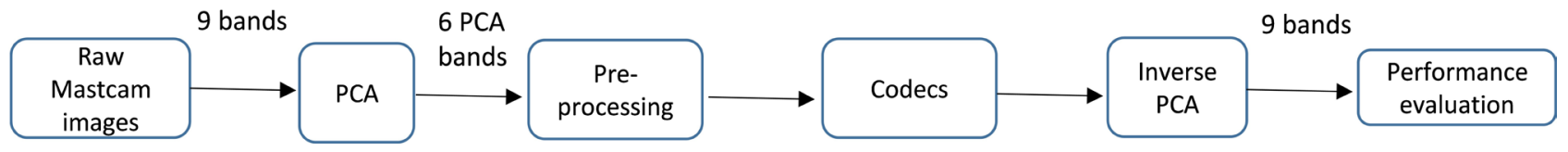

Figure 2. Two-step approach to compressing Mastcam images.

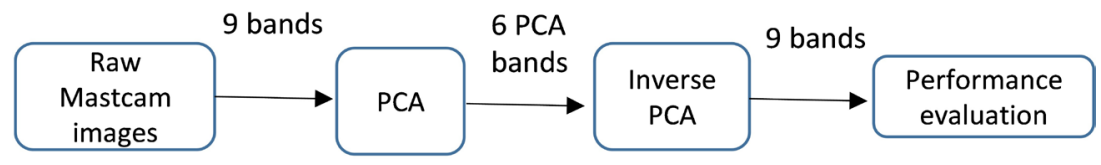

Figure 3. PCA only approach compressing Mastcam images. 
It is mainly used for still image compression.

- VP8 and VP9 [25] [26]:

These video compression algorithms are owned by Google. The performance is somewhat close to X-264. We did include VP8 and VP9 in our study because they are not as popular as X264 and X265.

- $\mathrm{X}-264$ [12]:

$\mathrm{X} 264$ is the current state-of-the-art in video compression. Youtube uses X264. It has good still image compression.

- X-265 [13]:

This is the next-generation video codec and has excellent still image compression and video compression. However, the computational complexity is much more than that of X264. In general, X265 has the same basic structure as previous standards and contains many incremental improvement over X264. It should be noted that X264 and X265 are optimized versions of H264 and H265, respectively.

- Daala [15]

Recently, there is a parallel activity at xiph.org foundation, which implements a compression codec called Daala [15]. It is based on DCT. There are pre- and post-filters to increase energy compaction and remove block artifacts. Daala borrows ideas from [14].

The block-coding framework in Daala can be illustrated in Figure 4 .

\section{Wavelet based algorithms}

JPEP-2000 is a wavelet [11] [27] [28] [29] based compression standard. It has better performance than JPEG. However, JPEG-2000 requires the use of the whole image for coding and hence is not suitable for real-time applications. In addition, motion-JPEG-2000 for video compression is not popular in the market.

\subsection{Performance Metrics}

In almost all compression systems, researchers used peak signal-to-noise ratio (PSNR) or structural similarity (SSIM) to evaluate the compression algorithms.

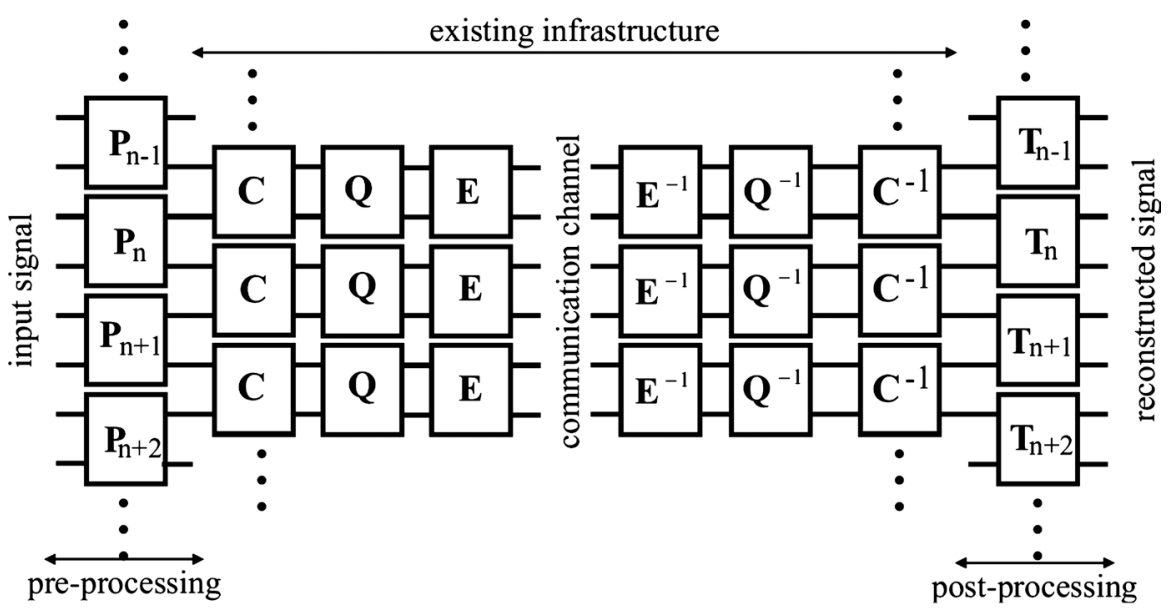

Figure 4. Daala codec for block-based image coding systems. 
Given a fixed compression ratio, algorithms that yield higher PSNR or SSIM will be regarded as better algorithms. However, PSNR or SSIM do not correlate well with human perception. Recently, a group of researchers investigated a number of different performance metrics [23]. Extensive experiments were performed to investigate the correlation between human perceptions with various performance metrics. According to the results found in [23], it was determined that two performance metrics correlate well with human perception. One image example shown in Figure 5 demonstrates that HVS and HVS-M have high correlation with human subjective evaluation results. In the past, we have used HVS and HVS-m in several applications [30] [31].

\section{Mastcam Image Compression Results}

\subsection{Mastcam Imager and Data}

Mastcam imager information is shown in Figure 6 and Table 1. There are 6 overlapping bands and 3 non-overlapping bands (L3, L4 and L5 from the left camera and R3, R4, and R5 from the right camera). More details about Mastcam can be found in [2].

\subsection{Mastcam Image Compression Results: Video Approach}

When using the Video approach for Daala, the nine-band image is saved as a three-frame video in the Y4M format. While Daala can also be used to encode still images, it was created with video compression as its primary use (Figure 7).

Figure 8 and Figure 9 show the comparison of different algorithms for 20 left and 20 right images, respectively. Table 2 and Table 3 summarize the performance metrics at 0.1 compression ratio for the left and right images, respectively. For left images, at 10 to 1 compression or 0.1 compression ratio, one can see that Daala is 5 and $10 \mathrm{~dB}$ better than that of JPEG in terms of HVS and HVSm, respectively. If we use the conventional metric (PSNR), then JPEG and J2K are
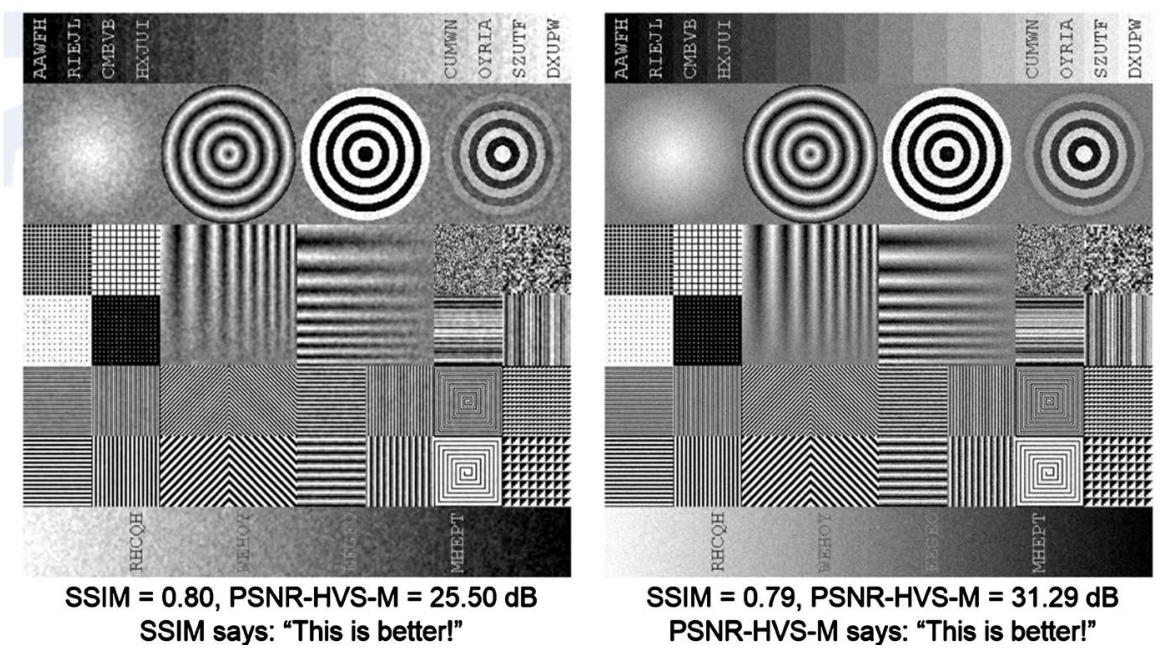

Figure 5. Comparison of SSIM and PSNR-HVS-M (HVSm). HVSm has better correlation with human perception [23]. 


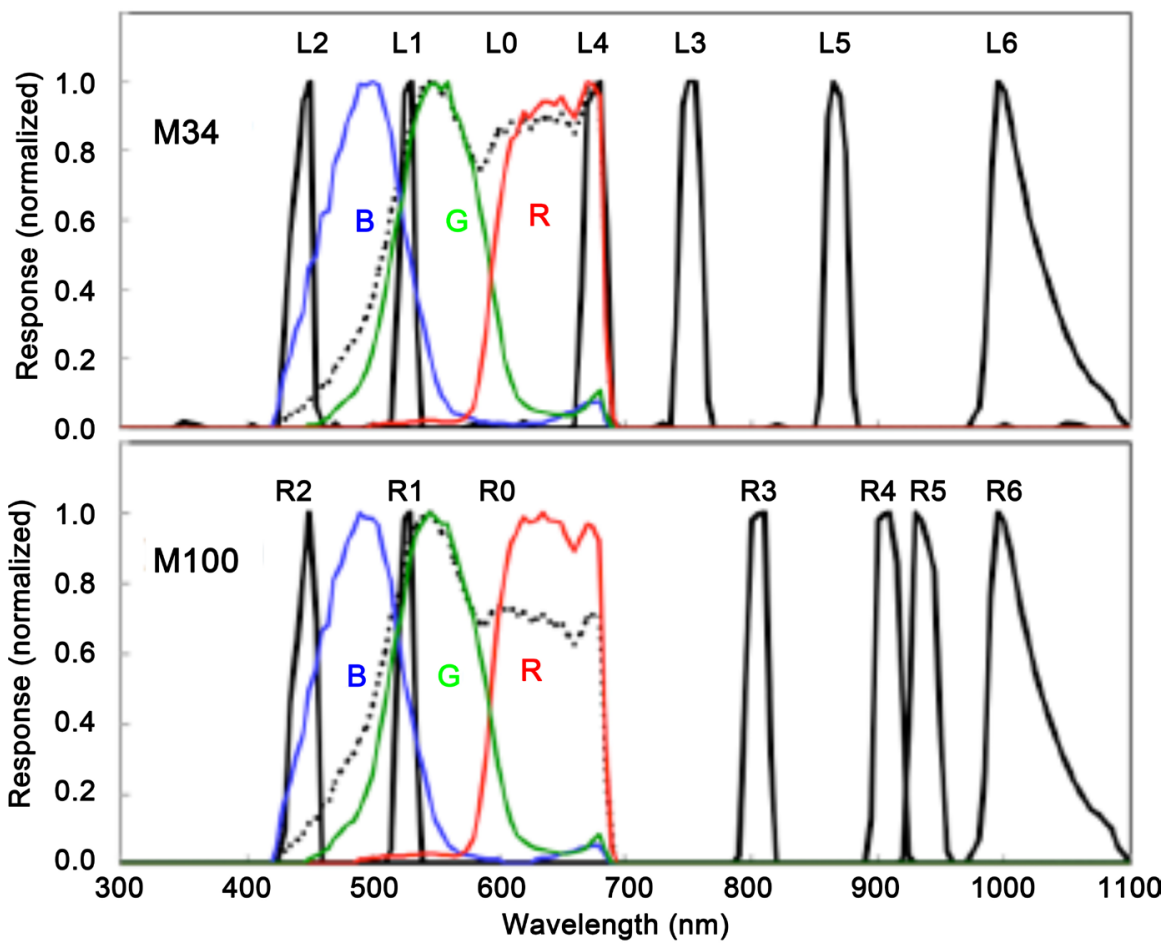

Figure 6. Normalized MSL/Mastcam system-level spectral response profiles for the left eye M-34 camera (top panel) and the right eye M-100 camera (bottom panel) [2].

Table 1. Mastcam filters in the order of increasing wavelengths [1].

\begin{tabular}{cccc}
\hline \multicolumn{2}{c}{ Left Mastcam } & \multicolumn{2}{c}{ Right Mastcam } \\
\hline Filter & Wavelength & Filter & Wavelength \\
\hline L2 & 445 & R2 & 447 \\
L0B & 495 & R0B & 493 \\
L1 & 527 & R1 & 527 \\
L0Gb & 554 & R0Gb & 551 \\
L0R & 640 & R0R & 638 \\
L4 & 676 & R3 & 805 \\
L3 & 751 & R4 & 908 \\
L5 & 867 & R5 & 937 \\
L6 & 1012 & R6 & 1013 \\
\hline
\end{tabular}

slightly better than others. In terms of SSIM, J2K and Daala are about 0.05 higher than that of the JPEG. For right images, the difference between Daala and JPEG is even bigger mainly because modern compression codecs are more efficient in compressing high resolution images.

Based on the results, we observe that Daala and J2K are very similar in performance. However when looking at the HVS and HVSm, Daala is the strongest method. 


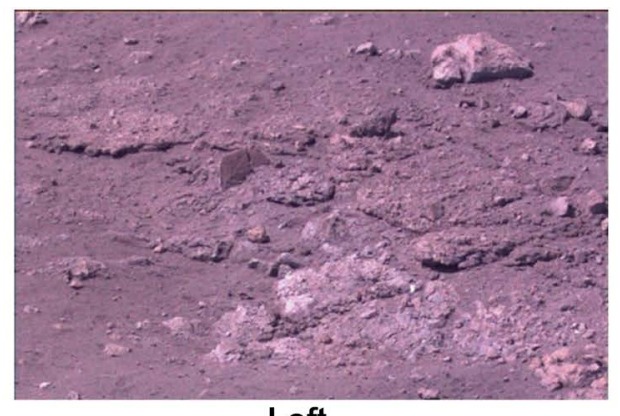

Left

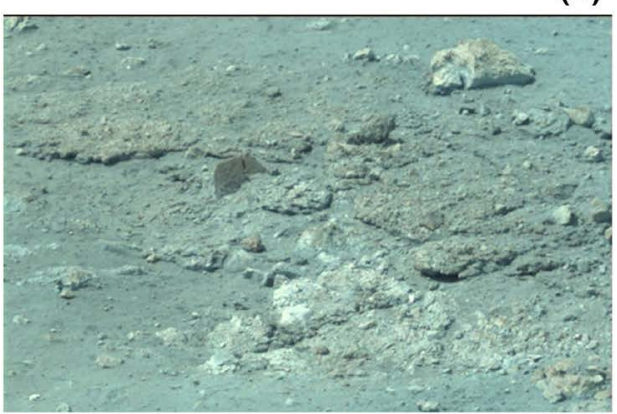

Left

(b)

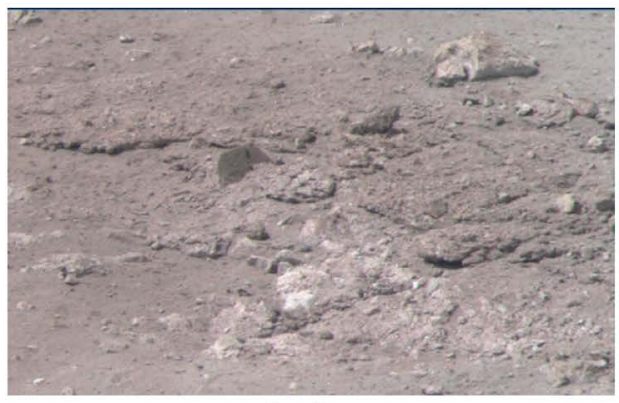

Left

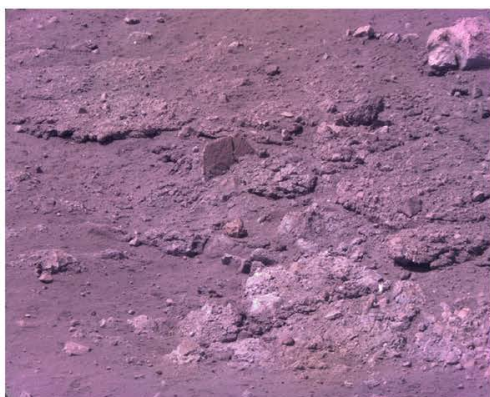

Right

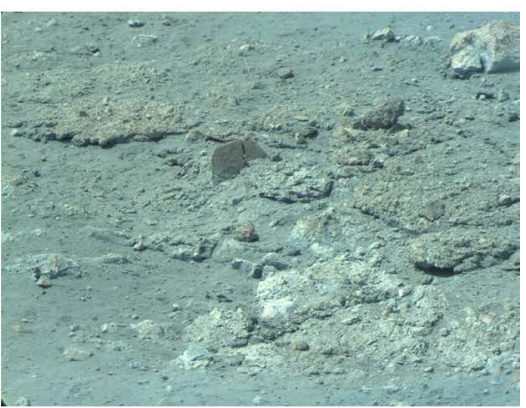

Right

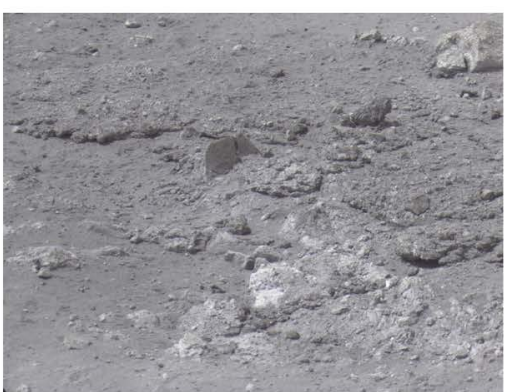

Right

(c)

Figure 7. Two exemplar 9-band Mastcam images (one left and one right). (a) Bands 1:3; (b) Bands 4:6; (c) Bands 7:9.

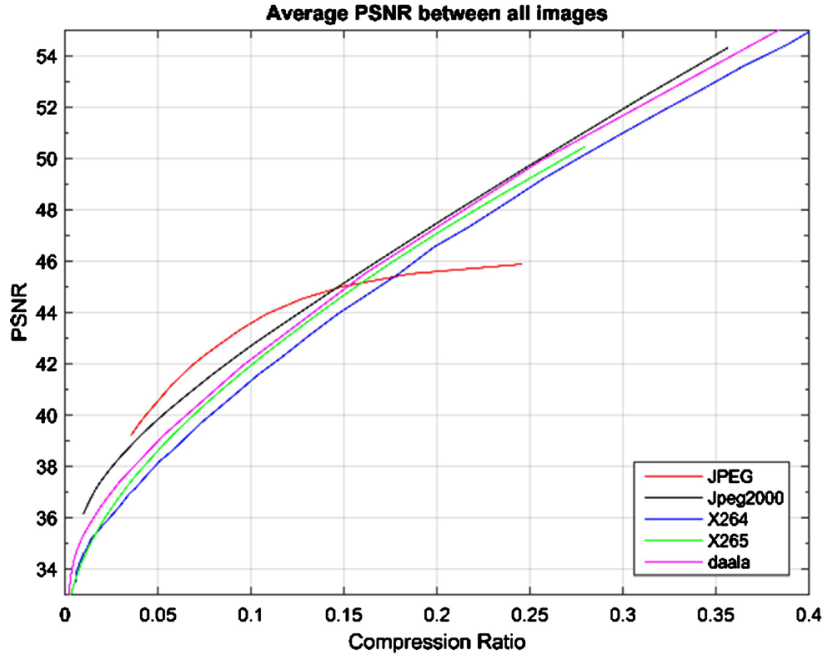

(a)

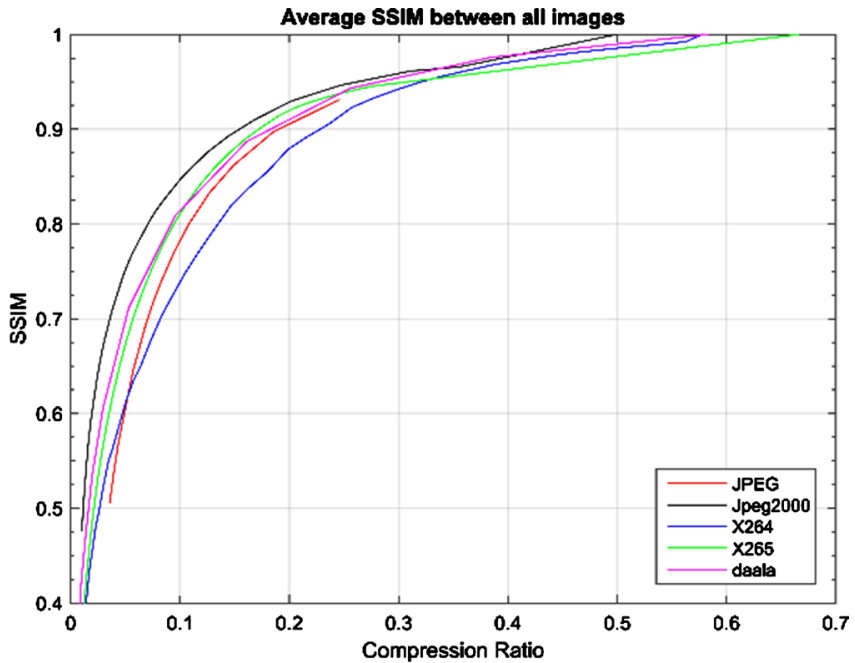

(b) 


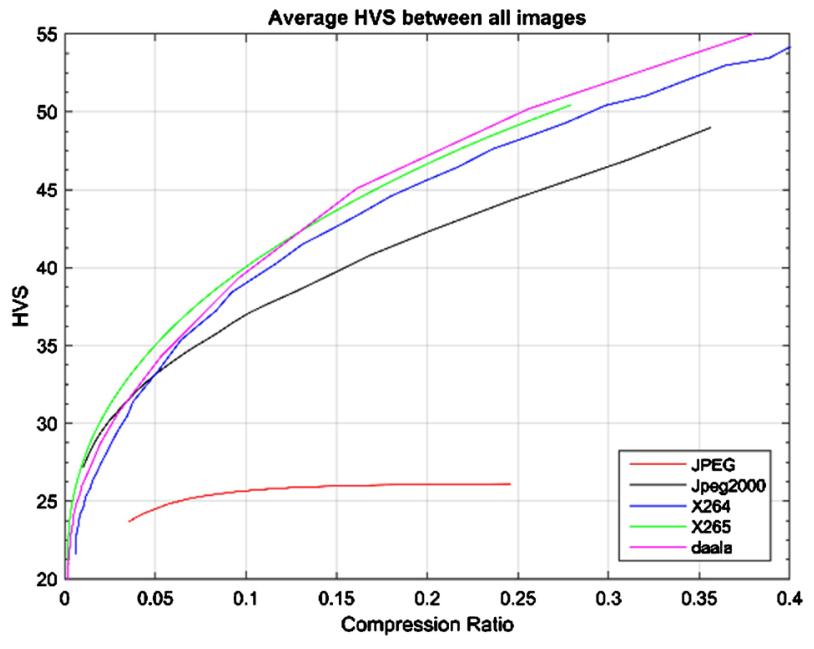

(c)

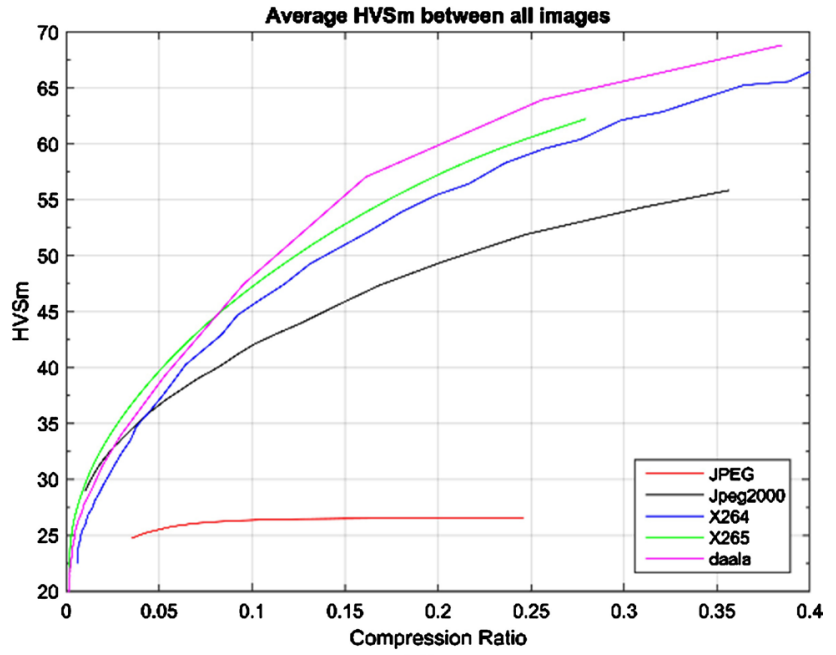

(d)

Figure 8. Comparison of different algorithms for 20 left images: (a) PSNR in dB for left images; (b) SSIM for left images; (c) HVS in $\mathrm{dB}$ for left images; (d) HVS-m in dB for left images.

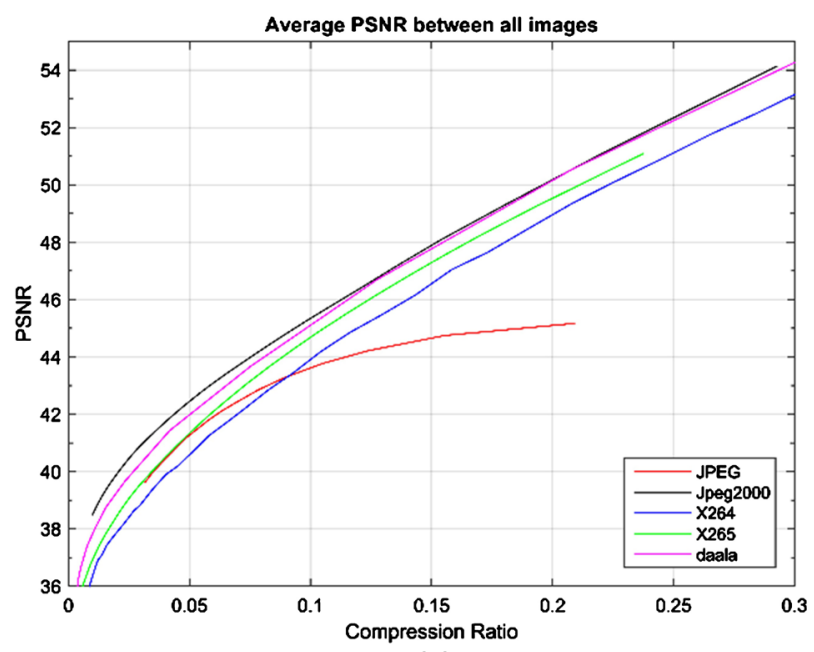

(a)

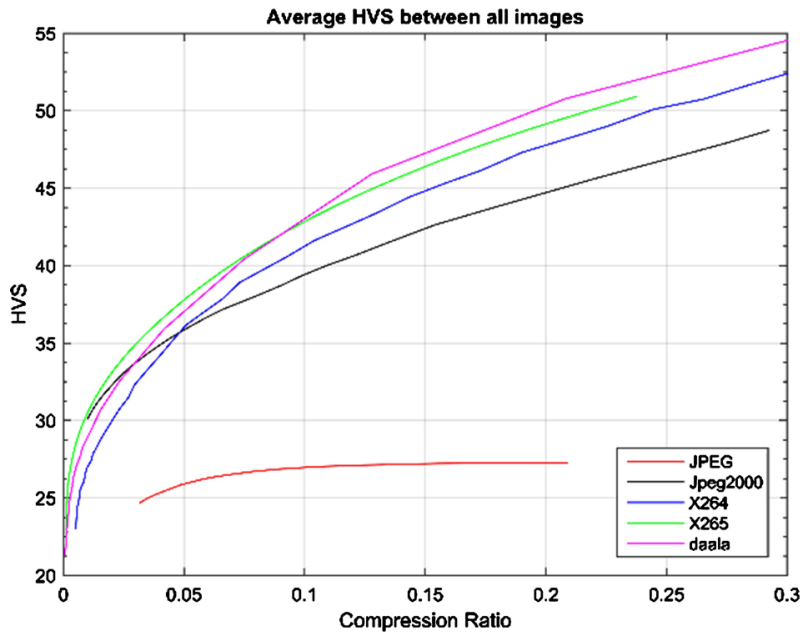

(c)

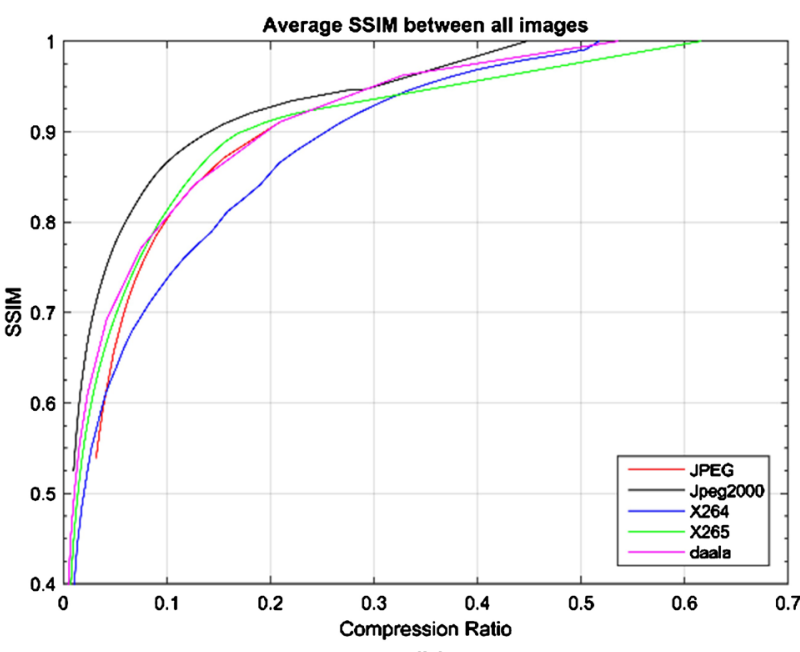

(b)

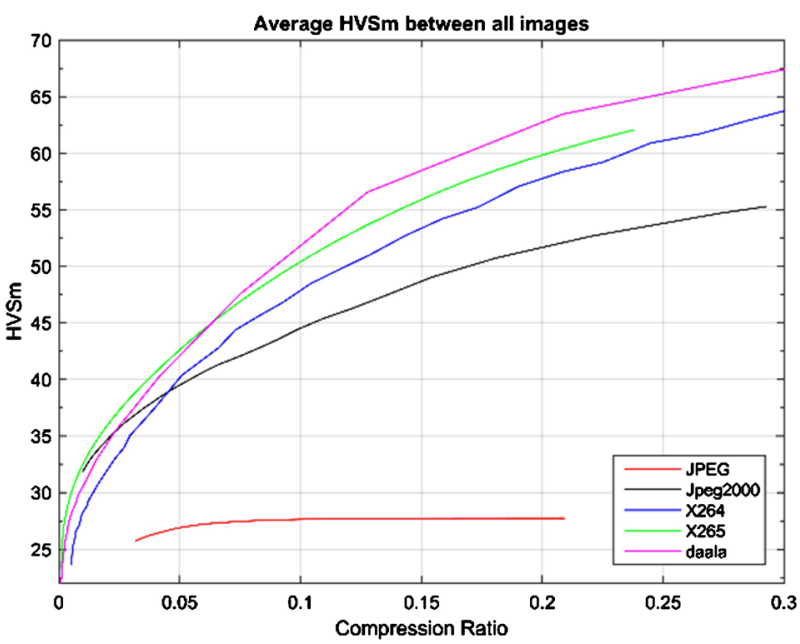

(d)

Figure 9. Comparison of different algorithms for 20 right images: (a) PSNR in dB for right images; (b) SSIM for right images; (c) HVS in $\mathrm{dB}$ for right images; (d) HVS-m in $\mathrm{dB}$ for right images. 
Table 2. Performance metrics of five codecs at 0.1 compression ratio for left Mastcam images. Bold numbers indicate the best performing method. Video approach.

\begin{tabular}{ccccc}
\hline & PSNR (dB) & SSIM & HVS (dB) & HVSm (dB) \\
\hline JPEG & $\mathbf{4 3 . 5}$ & 0.78 & 25.5 & 26 \\
J2K & 42.75 & $\mathbf{0 . 8 4}$ & 36.88 & 42 \\
X264 & 41.30 & 0.74 & 38 & 45.5 \\
X265 & 41.88 & 0.81 & $\mathbf{4 0}$ & 47 \\
Daala & 41.90 & 0.82 & 39.5 & $\mathbf{4 8}$ \\
\hline
\end{tabular}

Table 3. Performance metrics of five codecs at 0.1 compression ratio for right Mastcam images. Bold numbers indicate the best performing method. Video approach.

\begin{tabular}{ccccc}
\hline & PSNR (dB) & SSIM & HVS (dB) & HVSm (dB) \\
\hline JPEG & 43.5 & 0.8 & 27 & 27.5 \\
J2K & 45.3 & $\mathbf{0 . 8 6}$ & 38.75 & 44.38 \\
X264 & 44 & 0.74 & 41.3 & 48 \\
X265 & 44.6 & 0.81 & 43 & 50.3 \\
Daala & 45.1 & 0.80 & $\mathbf{4 3 . 5}$ & $\mathbf{5 1 . 8 7}$ \\
\hline
\end{tabular}

\subsection{Compression Using a Split Band (SB) Approach}

Figure 10, Figure 11, Table 4, and Table 5 show the different performance metrics using the SB approach. Let us focus on 0.1 compression ratio (10 times compression) for the left images first. For PSNR, J2K is about $3 \mathrm{dBs}$ better than that of JPEG. For SSIM, J2K and Daala are 0.06 and 0.04 better than JPEG. For HVS and HVSm, we can see that Daala's performance is 5 and $10 \mathrm{dBs}$ higher than that of JPEG, respectively. This means Daala's compression can preserve the perception quality in the reconstructed images.

In general, the performance metrics of right images are higher than those of the left images. The reason is that the modern codecs have better mechanisms to compress high resolution images.

\subsection{Compression Using PCA Only Approach}

PCA has been used as a compression tool in the past. It is also known as KLT (Kohonen Loeve Transform). Here, we briefly summarize the application of PCA only to compress left and right Mastcam images. Figure 12 and Figure 13 show that the compression performance of PCA3 and PCA6 is not enough to reach 10 to 1 compression. For instance, PCA3 achieved a PSNR of $41.25 \mathrm{dBs}$ at a compression ratio of 0.18 . We will see in Section 3.5 that it will be good to combine PCA with other codecs to further improve the compression performance to 10 to 1 .

\subsection{Compression Using a Two-Step Approach}

The first step performs the PCA compression. In the second step, we used the 


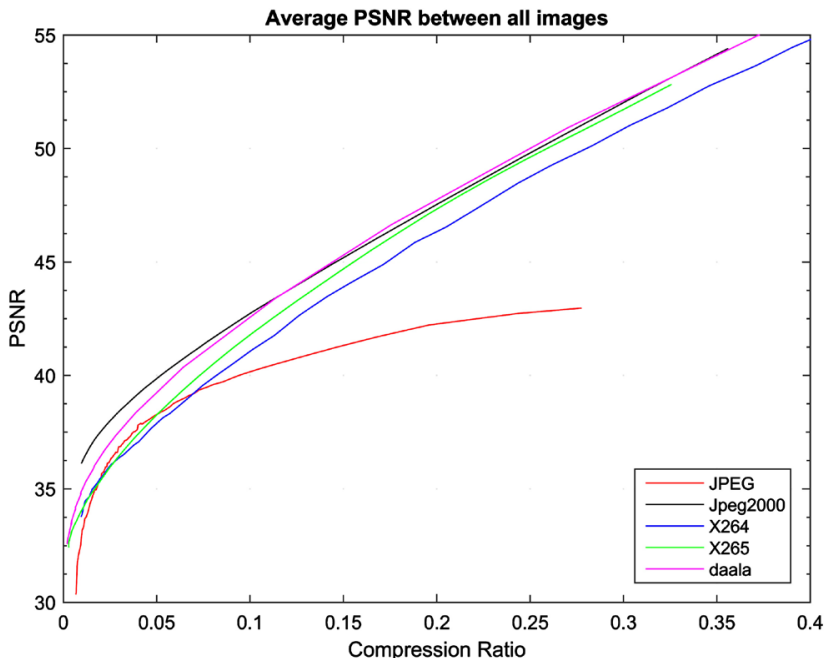

(a)

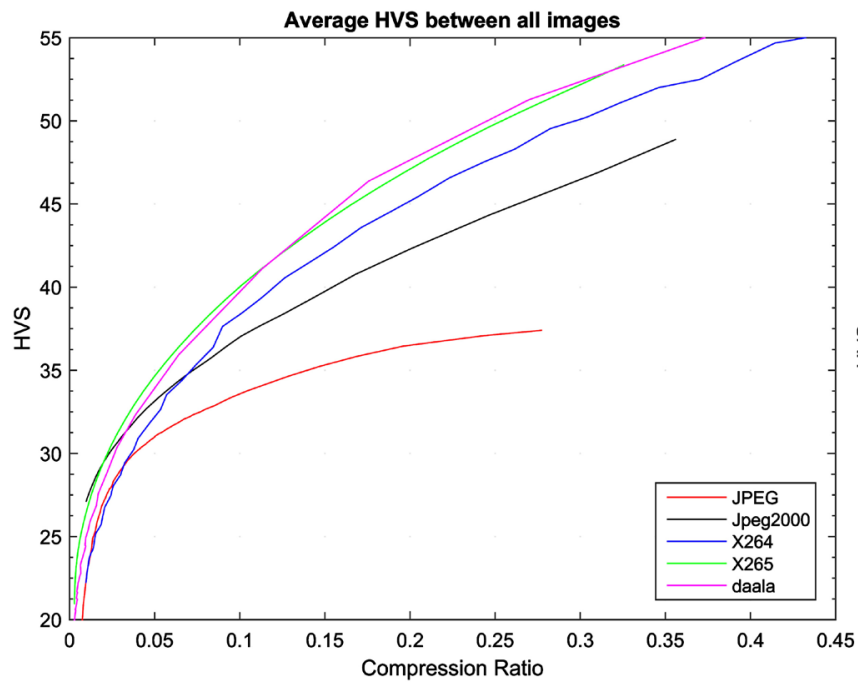

(c)

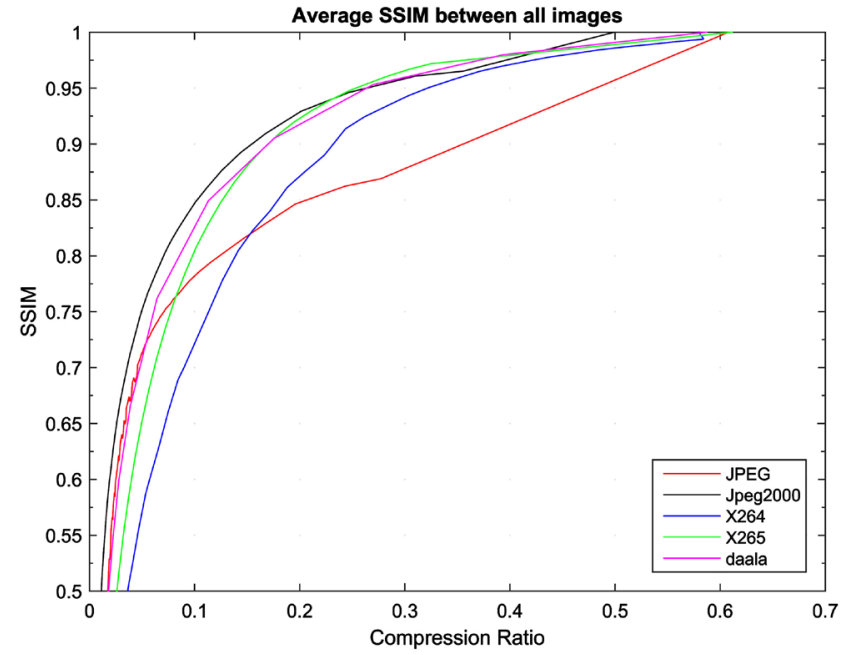

(b)

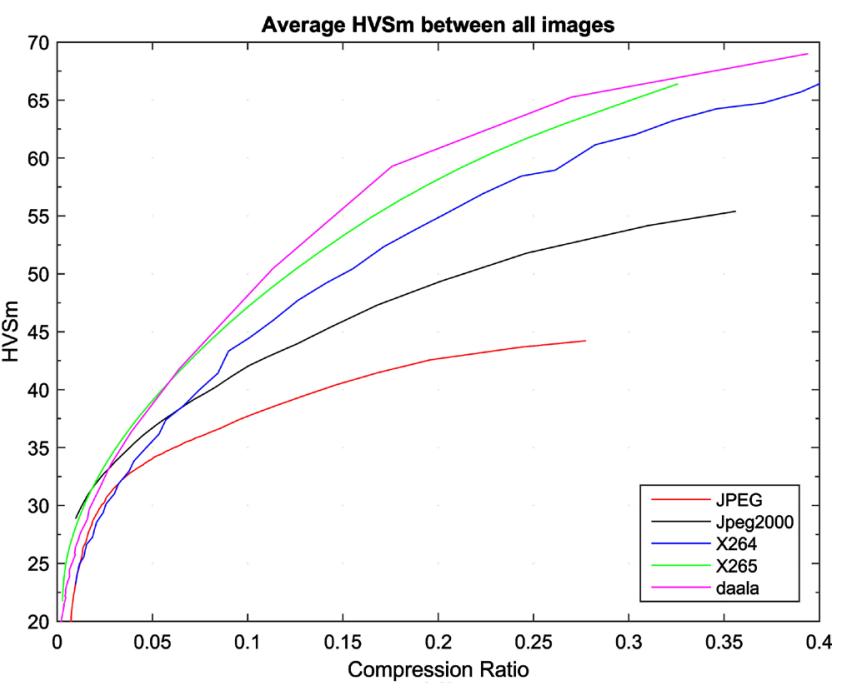

(d)

Figure 10. Comparison of different algorithms for 20 left images: (a) PSNR in dB for left images; (b) SSIM for left images; (c) HVS in $\mathrm{dB}$ for left images; (d) HVS-m in dB for left images.

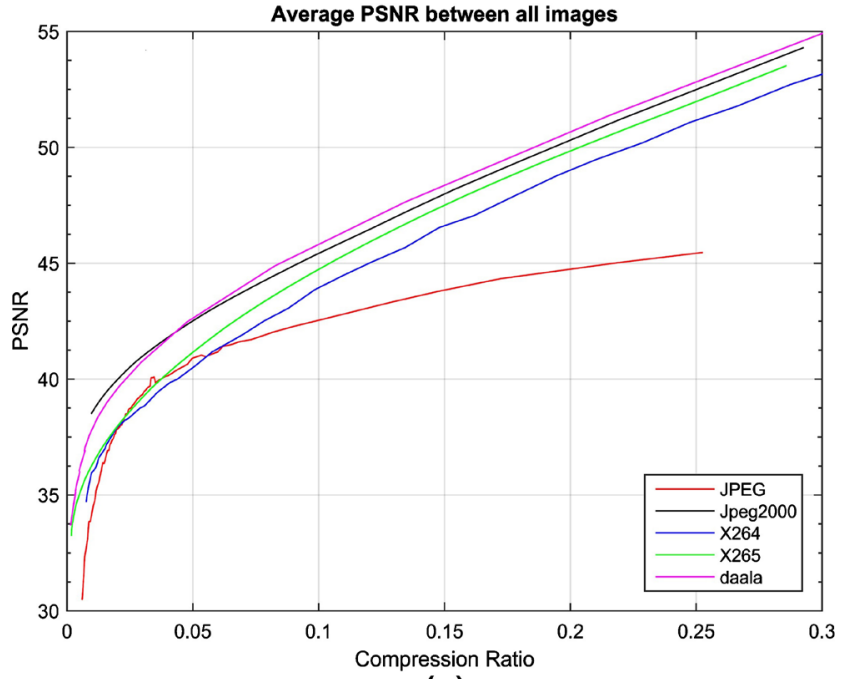

(a)

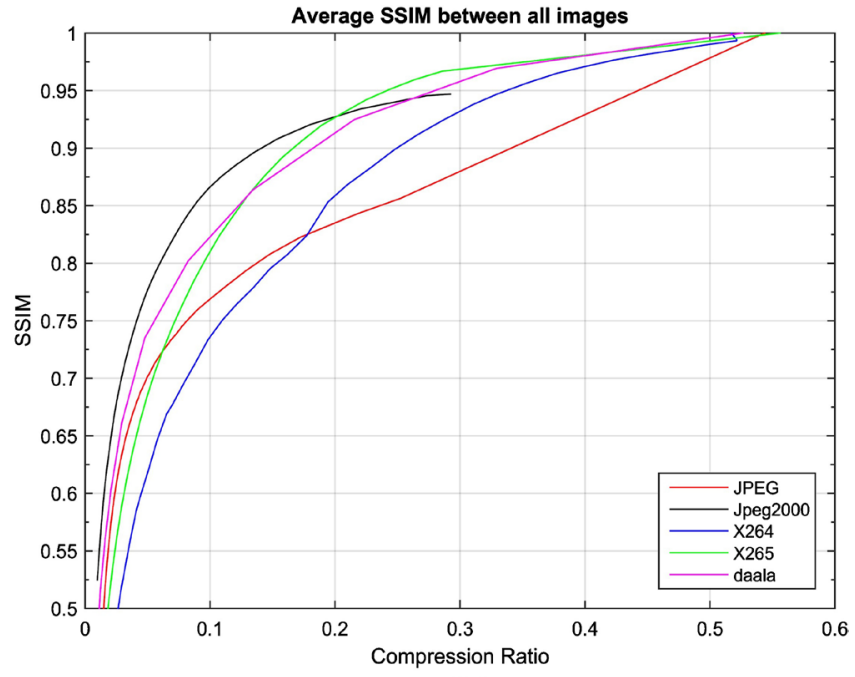

(b) 


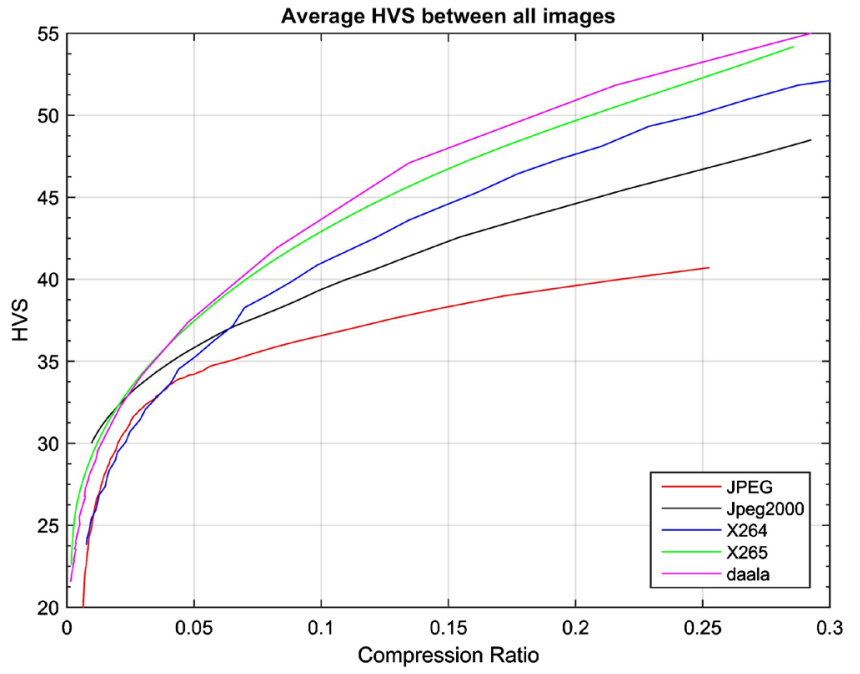

(c)

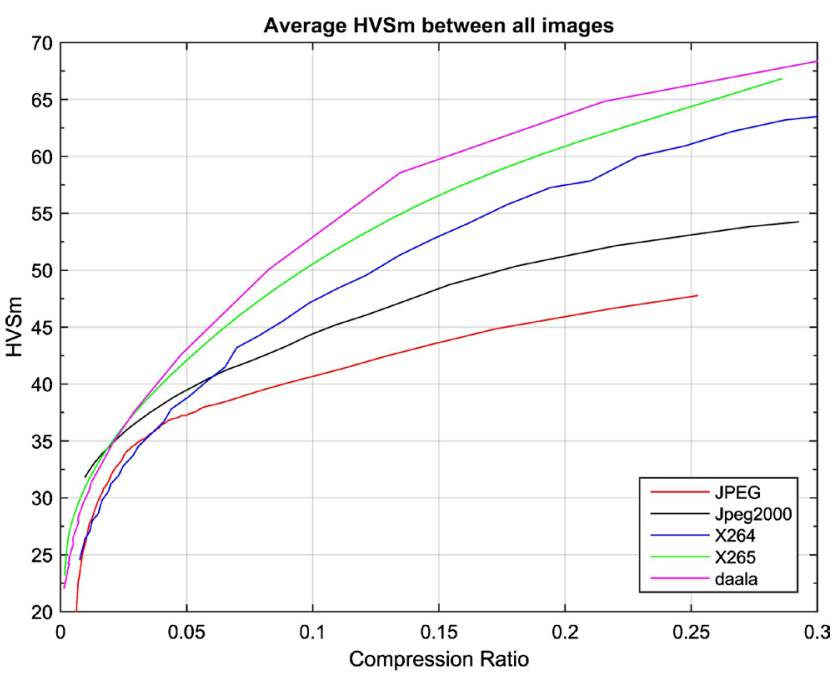

(d)

Figure 11. Comparison of different algorithms for 20 right images: (a) PSNR in dB for right images; (b) SSIM for right images; (c) HVS in $\mathrm{dB}$ for right images; (d) HVS-m in $\mathrm{dB}$ for right images.

Table 4. Performance metrics of five codecs at 0.1 compression ratio for left Mastcam images. Bold numbers indicate the best performing method. SB approach.

\begin{tabular}{ccccc}
\hline & PSNR (dB) & SSIM & HVS (dB) & HVSm (dB) \\
\hline JPEG & 40.15 & 0.79 & 33.7 & 37.5 \\
J2K & $\mathbf{4 2 . 7}$ & $\mathbf{0 . 8 5}$ & 36.88 & 41.25 \\
X264 & 41.05 & 0.73 & 38.25 & 44.37 \\
X265 & 41.87 & 0.81 & $\mathbf{4 0}$ & 47 \\
Daala & 42.5 & 0.83 & 39.5 & $\mathbf{4 8 . 1 2}$ \\
\hline
\end{tabular}

Table 5. Performance metrics of five codecs at 0.1 compression ratio for right Mastcam images. Bold numbers indicate the best performing method. SB approach.

\begin{tabular}{ccccc}
\hline & PSNR (dB) & SSIM & HVS (dB) & HVSm (dB) \\
\hline JPEG & 42.5 & 0.77 & 36.3 & 40.62 \\
J2K & 45.5 & $\mathbf{0 . 8 7}$ & 39.12 & 44.37 \\
X264 & 43.8 & 0.74 & 40.12 & 47.3 \\
X265 & 44.5 & 0.81 & 43 & 50.62 \\
Daala & $\mathbf{4 5 . 8}$ & 0.82 & $\mathbf{4 3 . 7 5}$ & $\mathbf{5 3}$ \\
\hline
\end{tabular}

video approach. In our earlier paper [16], we observed that PCA-6 is better than PCA-3. Here, we present new results for PCA-6 because: 1) we have included Daala results; 2) there are new updates of X265 and X264.

Figure 14, Table 6, Figure 15, and Table 7 show the performance metrics of the 2-step approach for left and right images. Let us focus again on the left images in the 0.1 compression ratio region. For PSNR, Daala is $6 \mathrm{dBs}$ better than that of JPEG. For SSIM, Daala is 0.16 higher than that of JPEG. For HVS and HVSm, Daala has 8 and $10 \mathrm{dBs}$ higher values than that of JPEG, respectively. For 


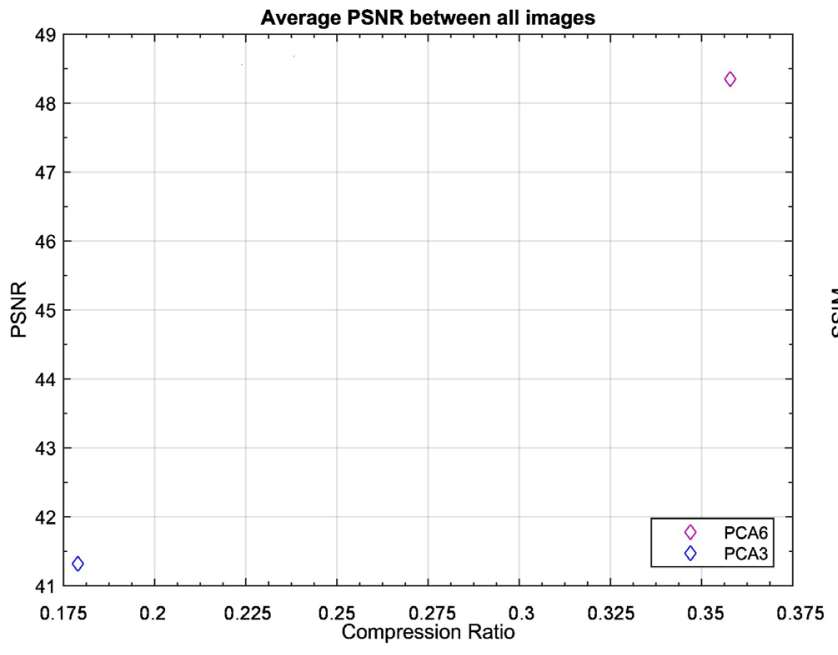

(a)

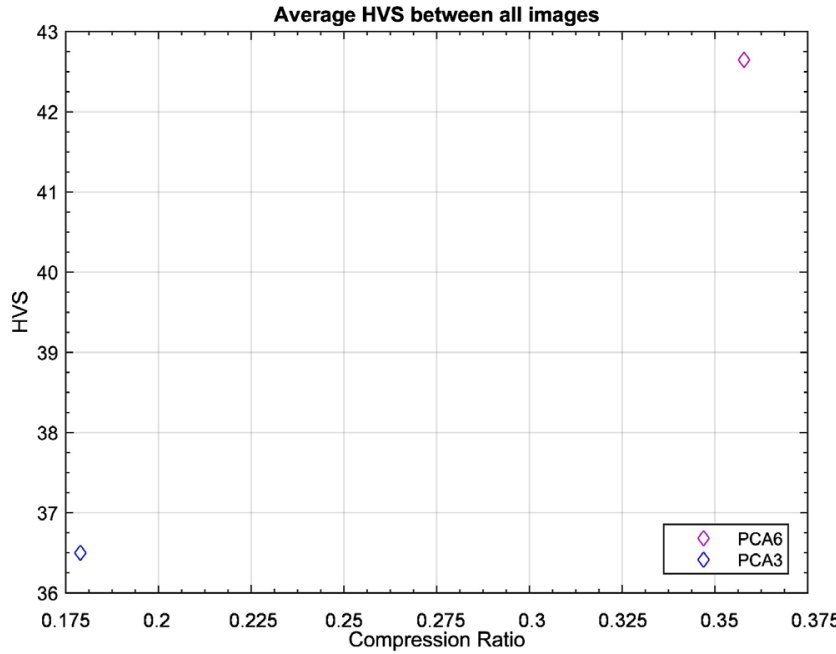

(c)

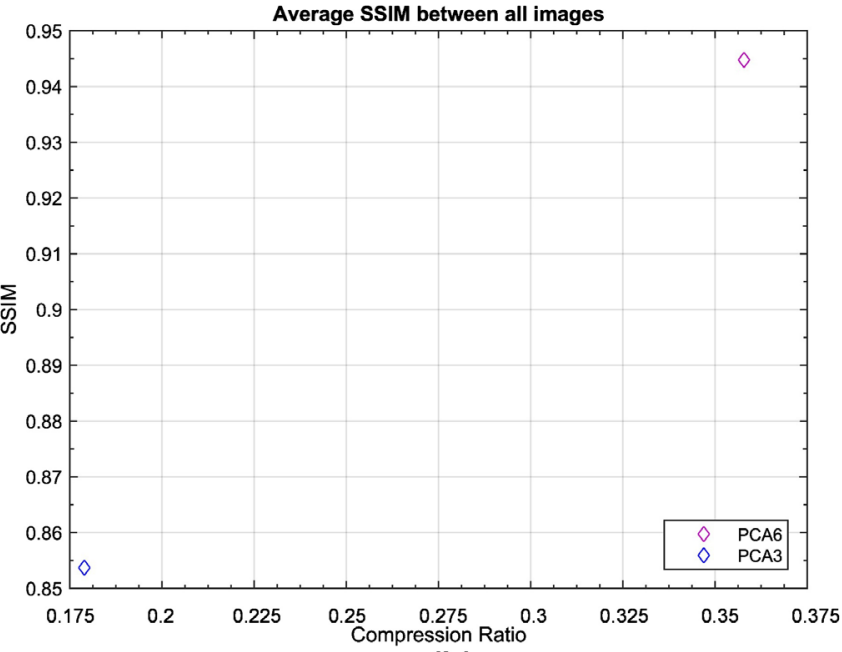

(b)

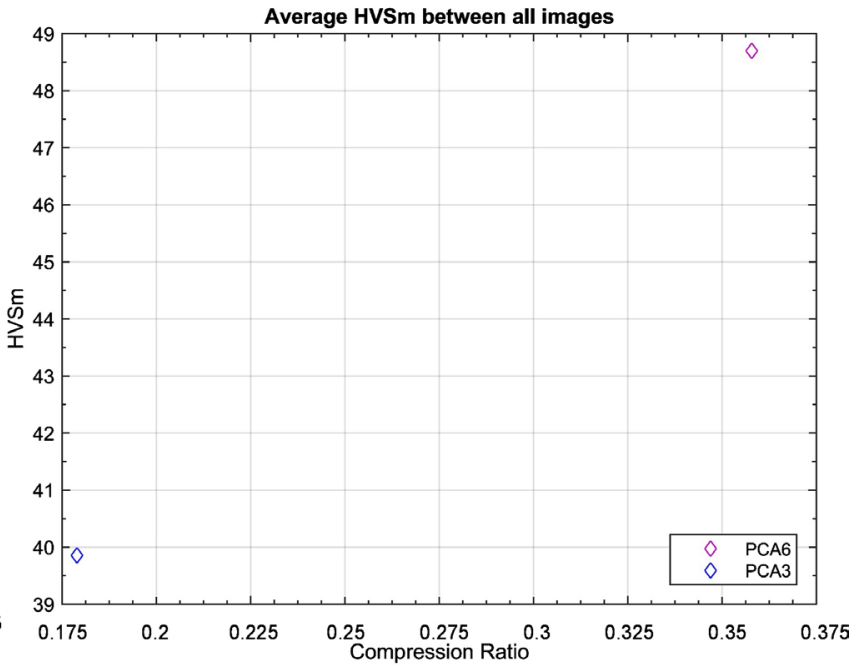

(d)

Figure 12. Comparison of different algorithms in the PCA only approach for 20 left images: (a) PSNR in dB for left images; (b) SSIM for left images; (c) HVS in dB for left images; (d) HVS-m in dB for left images.

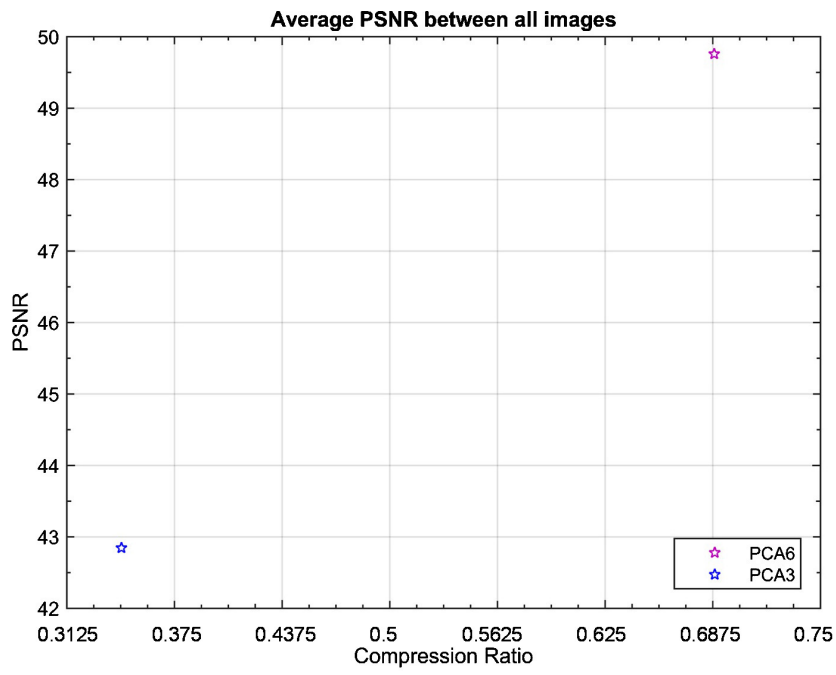

(a)

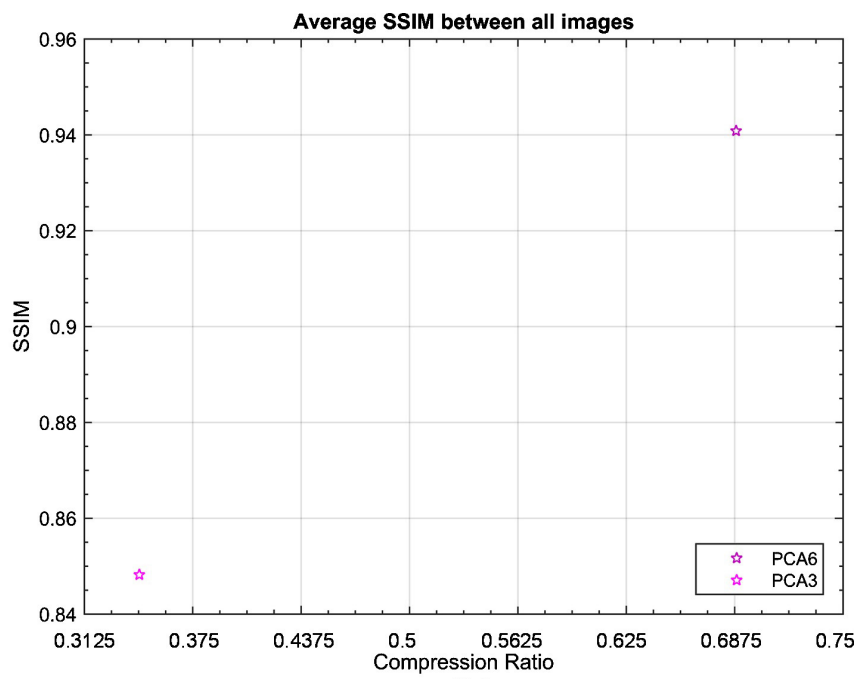

(b) 


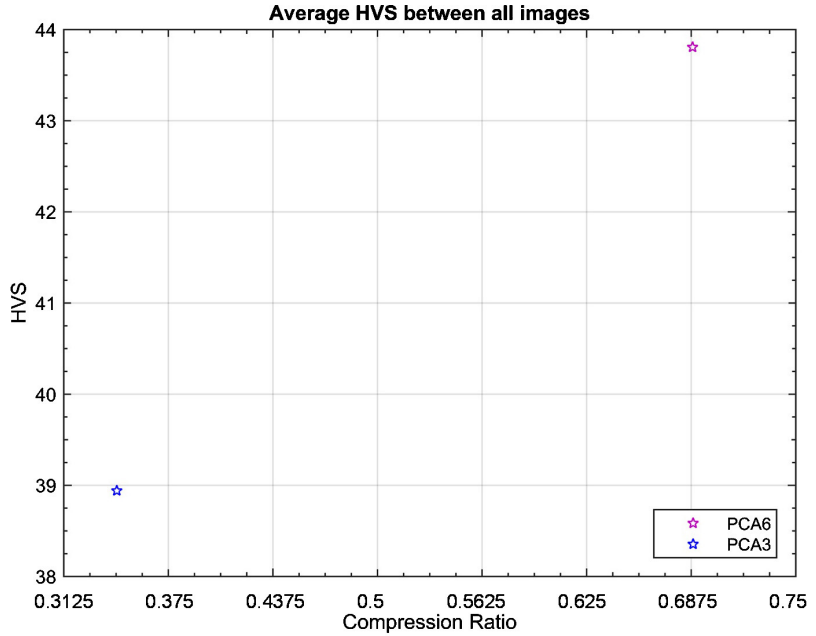

(c)

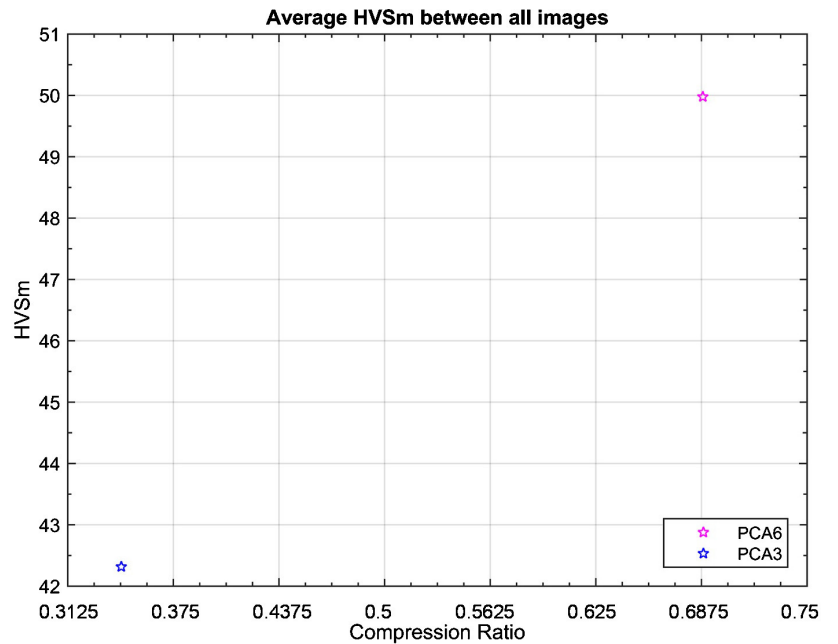

(d)

Figure 13. Comparison of different algorithms in the PCA only approach for 20 right images: (a) PSNR in dB for right images; (b) SSIM for right images; (c) HVS in dB for right images; (d) HVS-m in dB for right images.

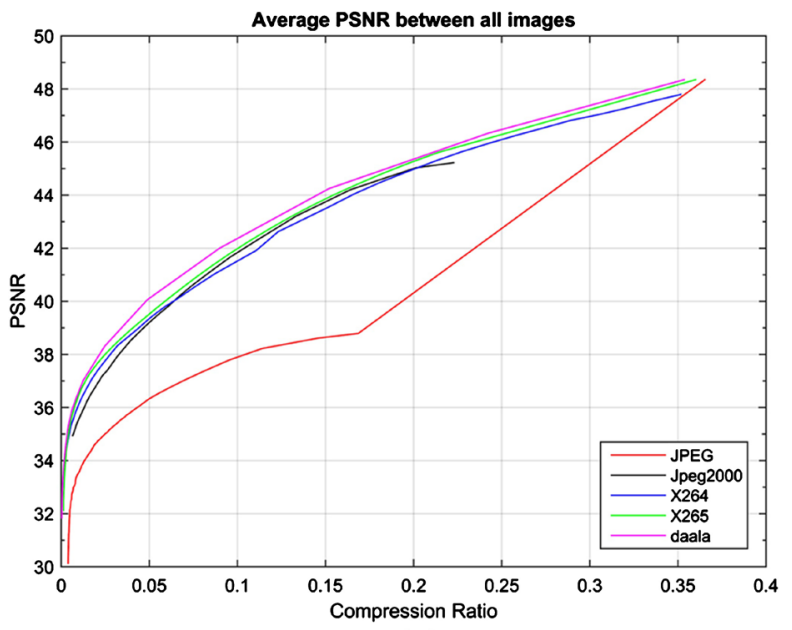

(a)

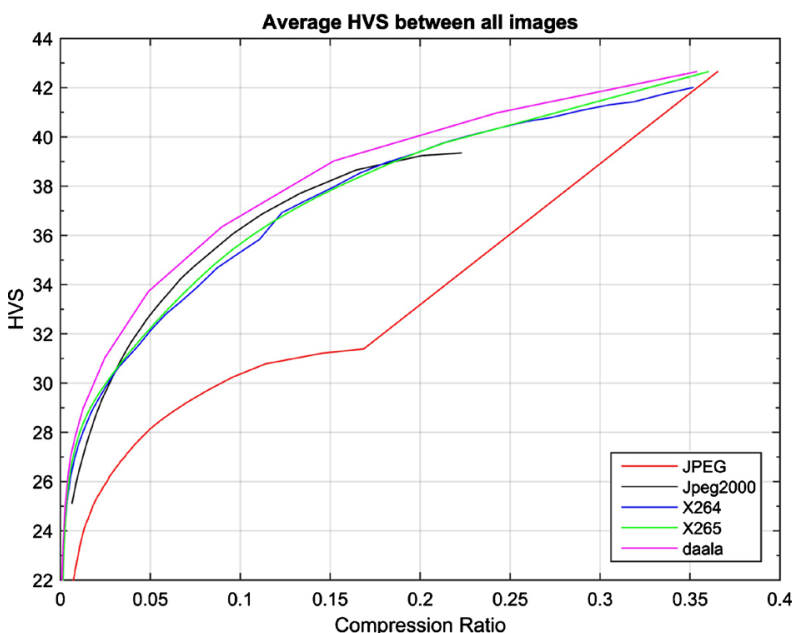

(c)

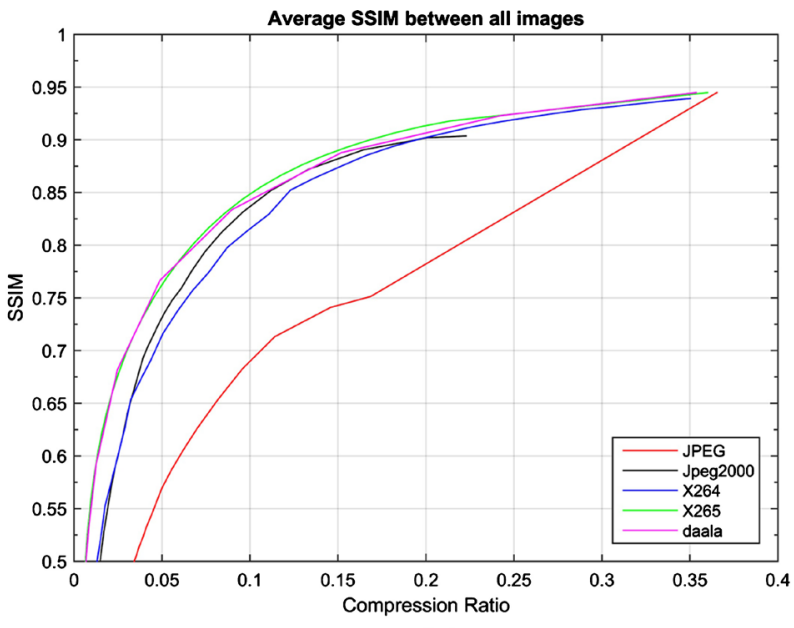

(b)

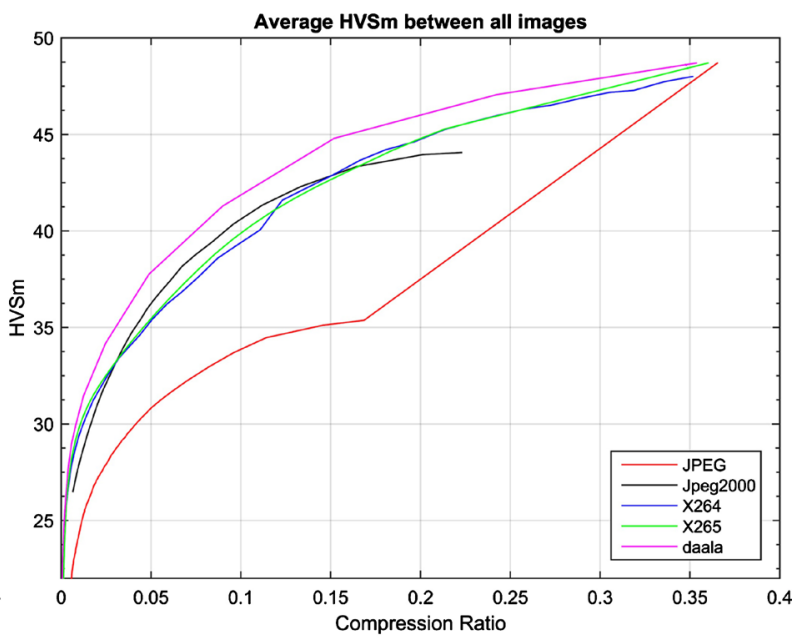

(d)

Figure 14. Comparison of different algorithms in the 2-step approach for 20 left images: (a) PSNR in dB for left images; (b) SSIM for left images; (c) HVS in dB for left images; (d) HVS-m in dB for left images. Here, PCA is used to compress 9 bands to 6 bands. 


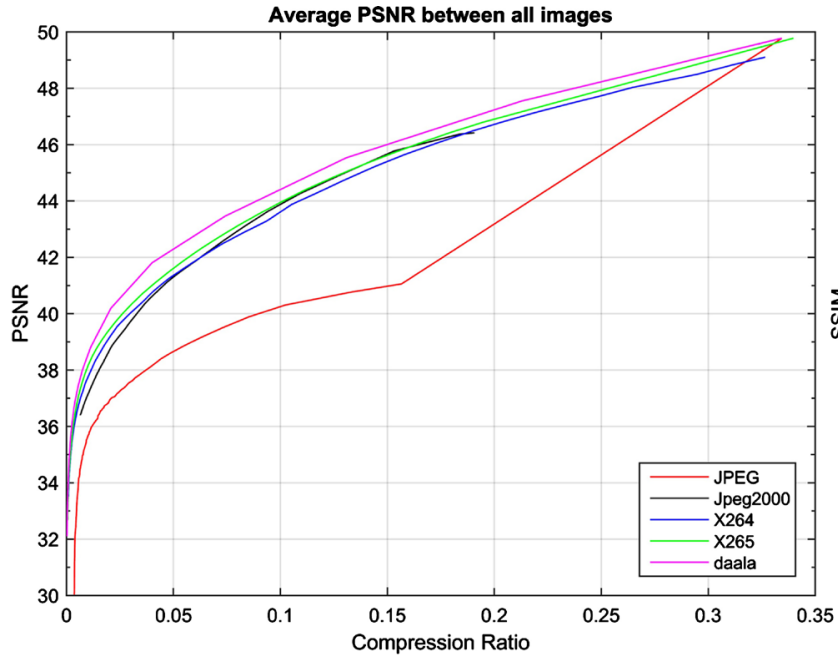

(a)

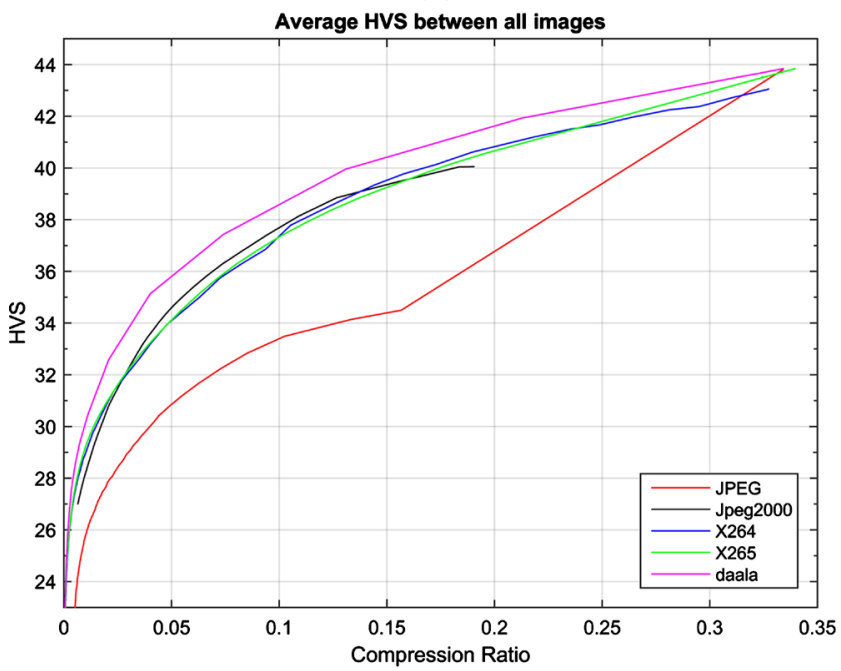

(c)

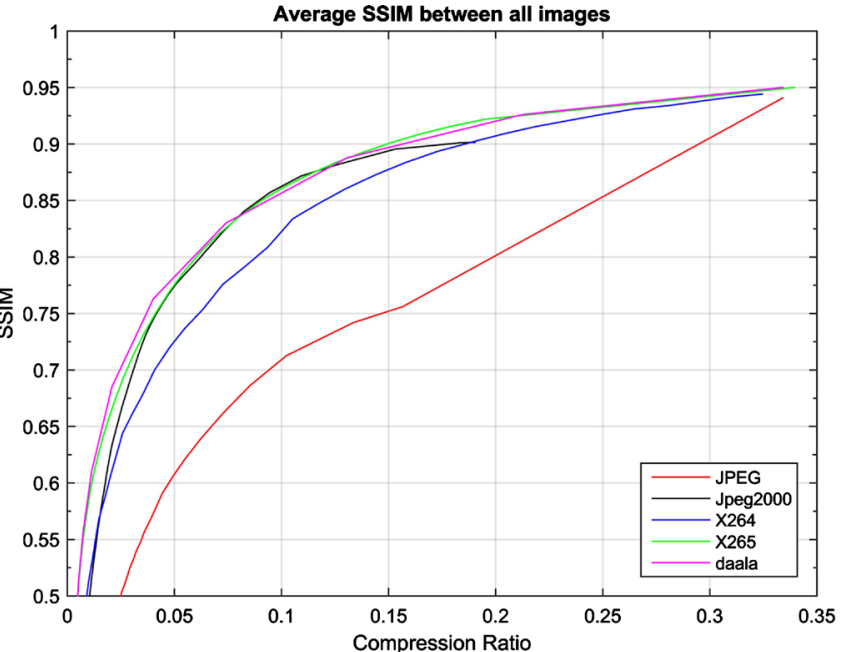

(b)

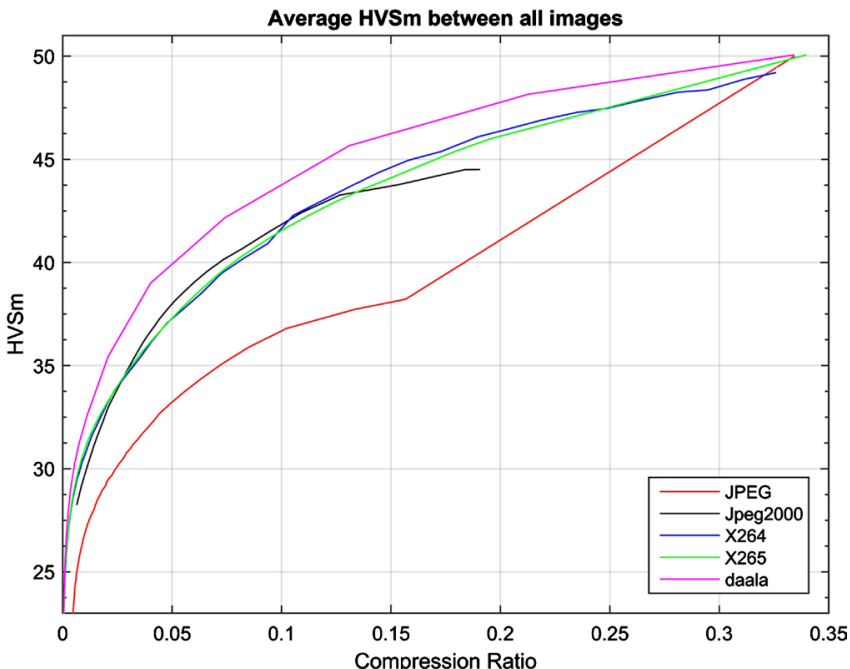

(d)

Figure 15. Comparison of different algorithms in the 2-step approach for 20 right images: (a) PSNR in dB for right images; (b) SSIM for right images; (c) HVS in dB for right images; (d) HVS-m in dB for right images. Here, PCA is used to compress 9 bands to 6 bands.

Table 6. Performance metrics of five codecs at 0.1 compression ratio for left Mastcam images. Bold numbers indicate the best performing method. Two-step approach.

\begin{tabular}{ccccc}
\hline & PSNR (dB) & SSIM & HVS (dB) & HVSm (dB) \\
\hline JPEG & 37.88 & 0.68 & 30.3 & 33.8 \\
J2K & 41.75 & 0.84 & 36.25 & 40.63 \\
X264 & 41.6 & 0.81 & 35.25 & 38.75 \\
X265 & 42 & $\mathbf{0 . 8 5}$ & 35.6 & 40 \\
Daala & $\mathbf{4 2 . 3 8}$ & 0.845 & $\mathbf{3 6 . 7 5}$ & $\mathbf{4 1 . 8 8}$ \\
\hline
\end{tabular}

right images, the differences between Daala and JPEG are even bigger.

\subsection{Comparison between Video and SB Approaches}

Now, we would like to compare the Video and SB approaches. Figure 16 and 
Figure 17 show the detailed comparison between video and the SB approaches for the left and right images, respectively. Let us first focus on the left images near the compression rate of 0.1 . We have the following observations:

Table 7. Performance metrics of five codecs at 0.1 compression ratio for left Mastcam images. Bold numbers indicate the best performing method. Two-step approach.

\begin{tabular}{ccccc}
\hline & PSNR (dB) & SSIM & HVS (dB) & HVSm (dB) \\
\hline JPEG & 40.25 & 0.71 & 33.5 & 36.85 \\
J2K & 43.8 & 0.862 & 37.7 & 41.88 \\
X264 & 43.6 & 0.82 & 37.4 & 41.56 \\
X265 & 43.9 & 0.861 & 37.4 & 41.55 \\
Daala & $\mathbf{4 4 . 3 8}$ & 0.86 & $\mathbf{3 8 . 6}$ & $\mathbf{4 3 . 7 5}$ \\
\hline
\end{tabular}

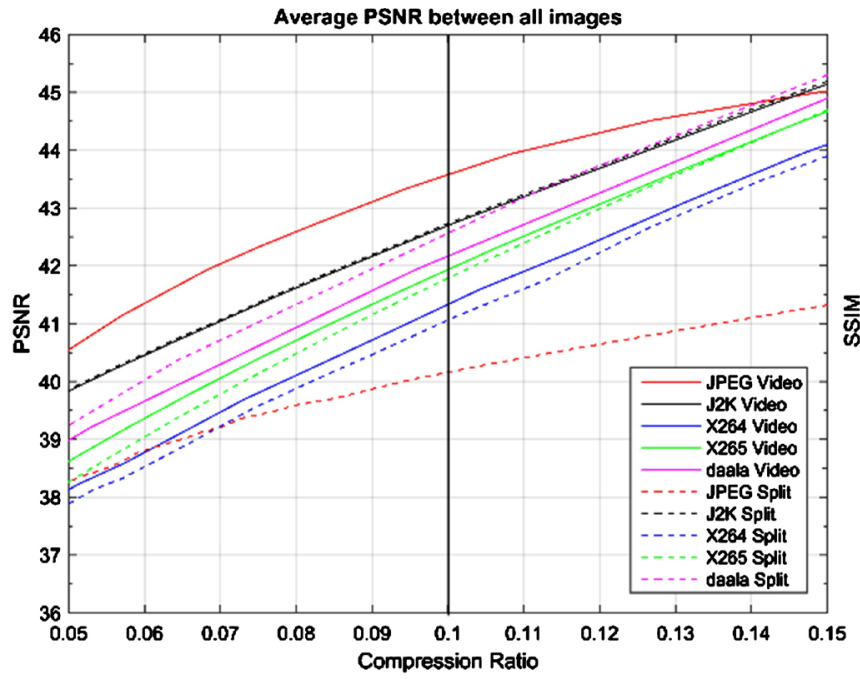

(a)

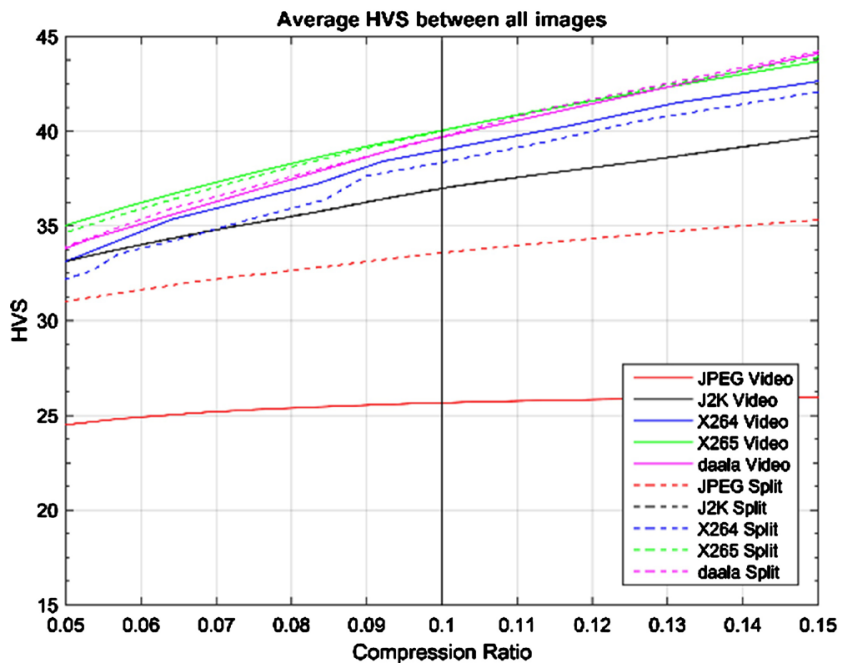

(c)

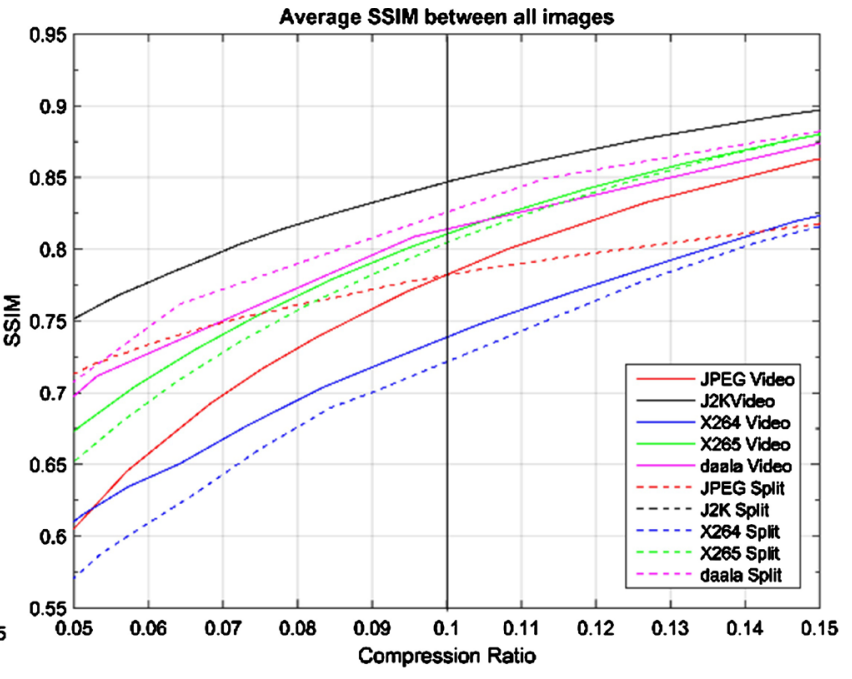

(b)

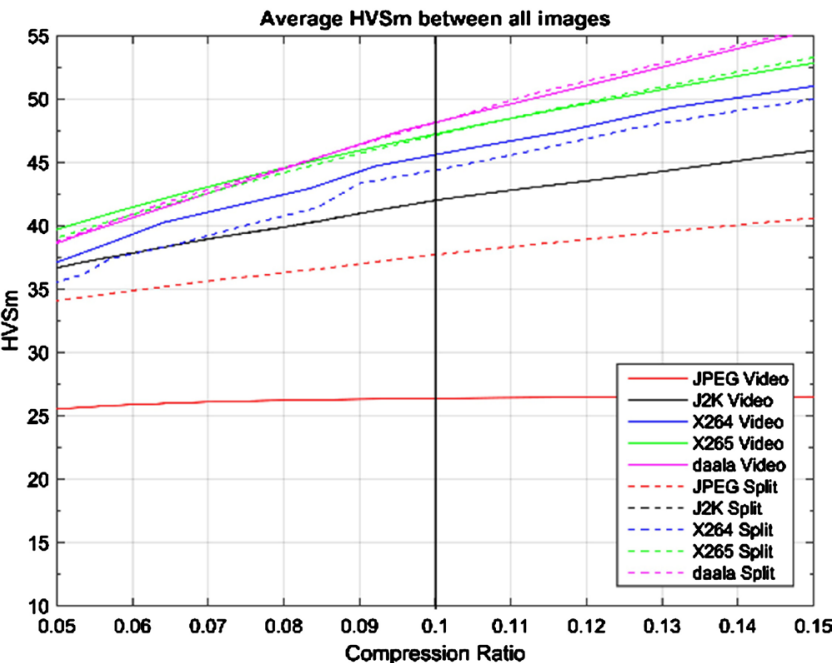

(d)

Figure 16. Comparison of video and SB approaches for 20 left images near the 0.1 compression ratio region. (a) Averaged PSNR in $\mathrm{dB}$ of left images; (b) Averaged SSIM of left images; (c) Averaged HVS in dB of left images; (d) Averaged HVS-m in dB of left images. 


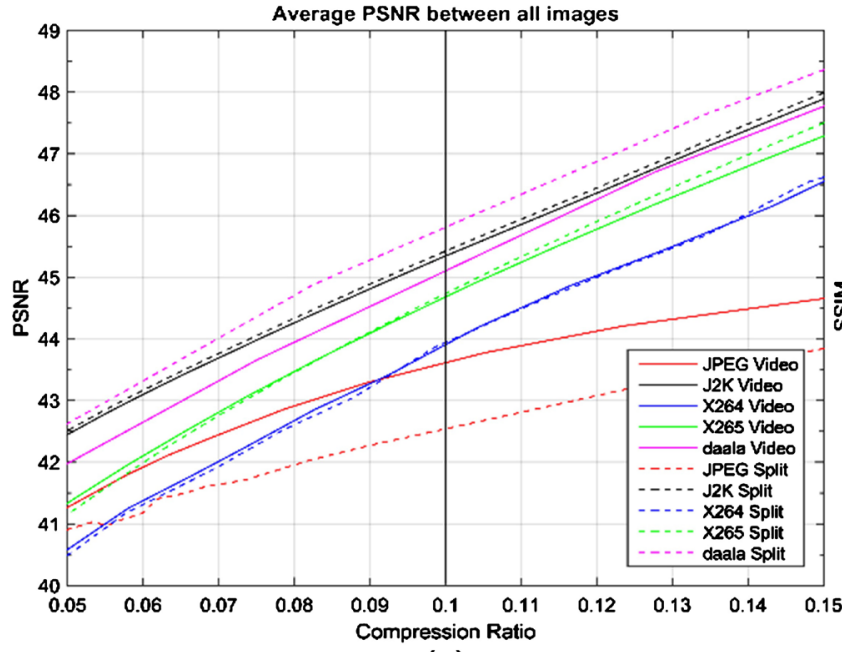

(a)

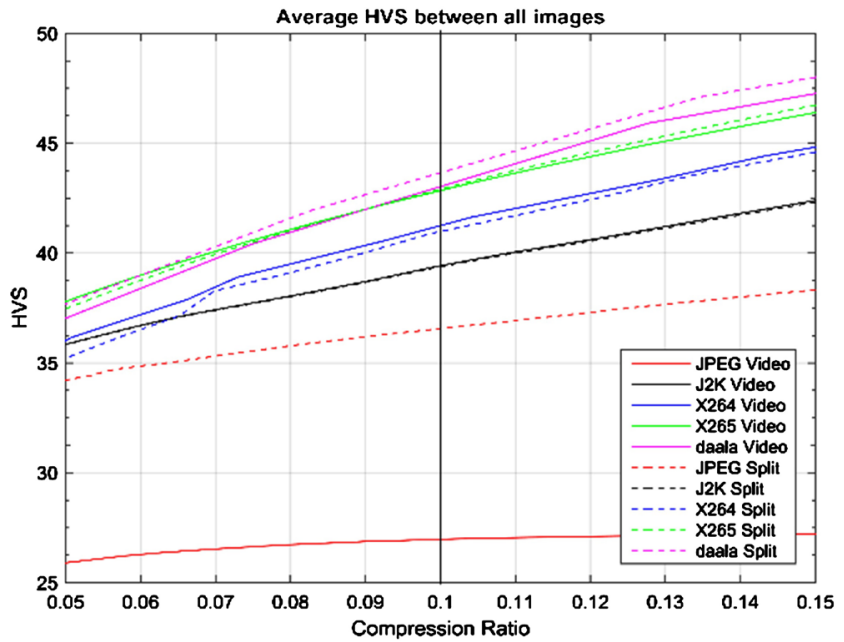

(c)

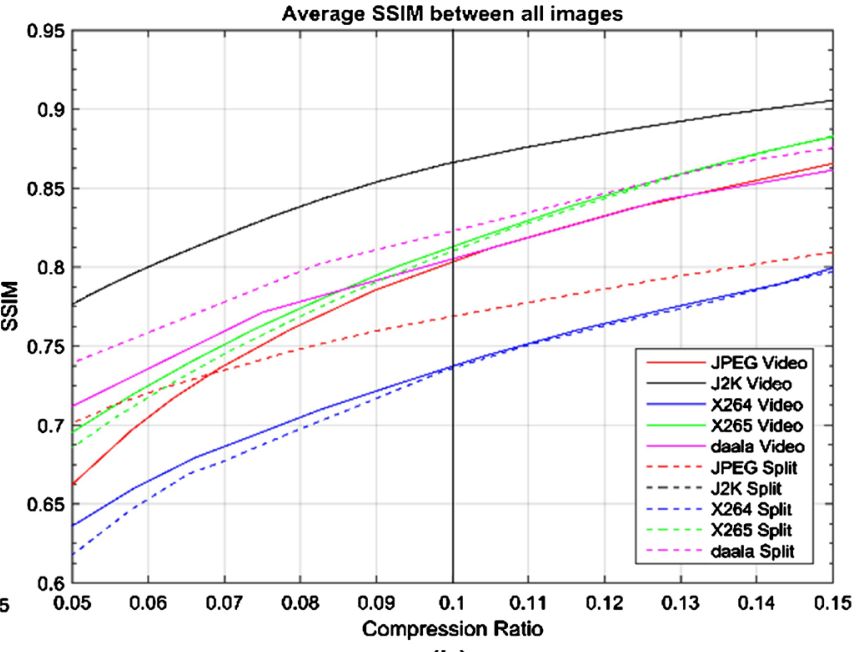

(b)

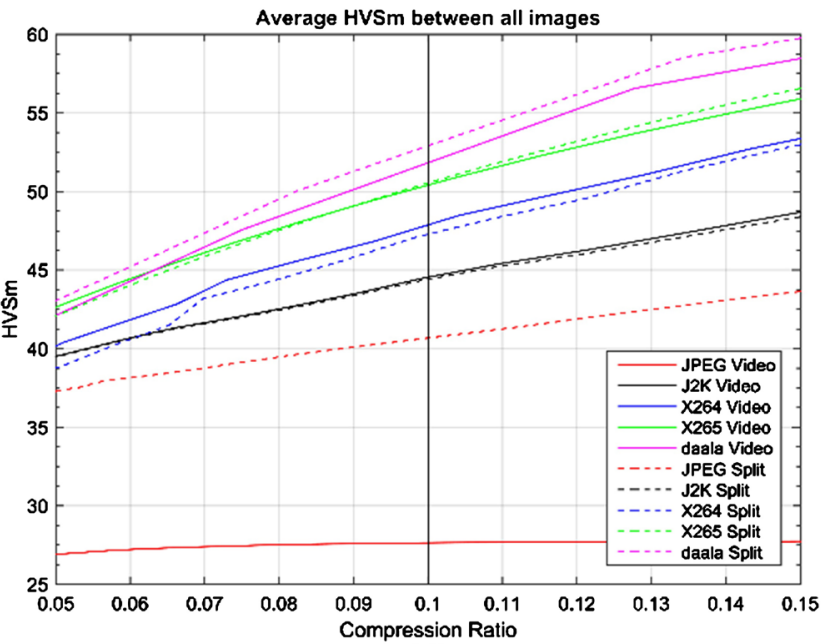

(d)

Figure 17. Comparison of video and SB approaches for 20 right images near the 0.1 compression ratio region. (a) Averaged PSNR in $\mathrm{dB}$ of right images; (b) Averaged SSIM of right images; (c) Averaged HVS in dB of right images; (d) Averaged HVS-m in dB of right images.

- JPEG has the highest PSNR than others.

- Daala has a very high HVSm ( $>45 \mathrm{~dB})$ in both video and SB approaches.

- Daala SB is slightly stronger than the Video approach.

- Daala SB produces a PSNR that is slightly weaker than J2K.

- Daala SB Produces a stronger SSIM than video but both approaches are below $\mathrm{J} 2 \mathrm{~K}$.

For right images, we see a similar trend as above except that the metrics are all higher in right images.

Hence, video approach is slightly worse than SB because video has more overhead and there are only three frames. If there are more frames, the Video approach may have edges over the SB approach. Since our interest is to achieve perceptually lossless compression, Daala is a better choice than others. In addition, Daala is amenable to parallel processing whereas J2K requires the whole image for processing and is not suitable for parallel implementation. 


\subsection{Comparison between the Video and Two-Step Approaches}

Here, we would like to compare the video and the 2-step approaches. Figure 18 and Figure 19 show the detailed comparison between Video and the 2-step approaches for the left and right images, respectively. Let us first focus on the left images near the compression rate of 0.1. In terms of HVS and HVSm, the Video approach with Daala is better than others most of the time. The Daala's performance is $7 \mathrm{~dB}$ and $10 \mathrm{~dB}$ better than JPEG in HVS and HVSm, respectively. X265 is the second best in HVS and HVSm. Finally, all the metrics in right images are higher than those corresponding left ones.

\subsection{Compression of RGB Images Only}

Figure 20, Figure 21, Table 8, and Table 9 show the metrics of different codecs for left and right RGB images, respectively. It can be seen that, at 0.1 compression

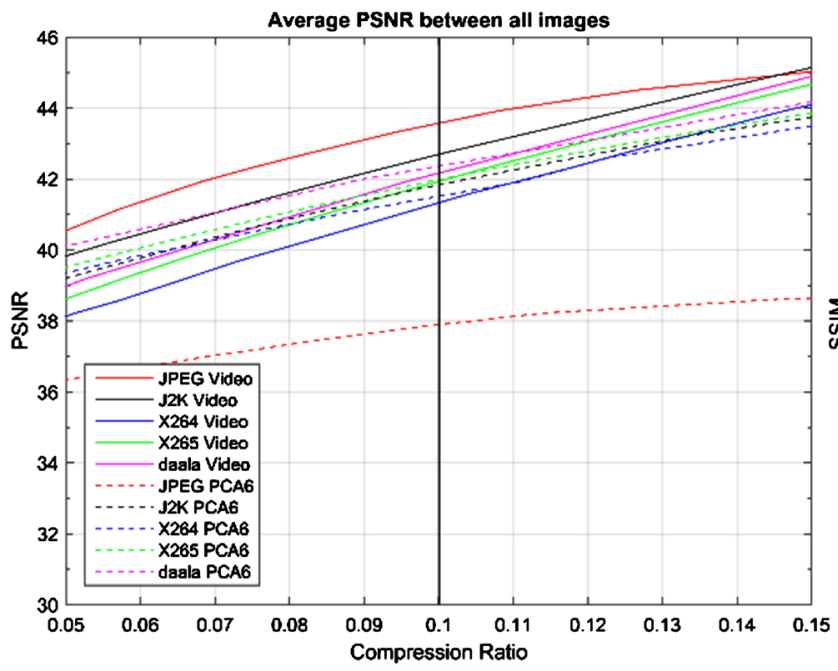

(a)

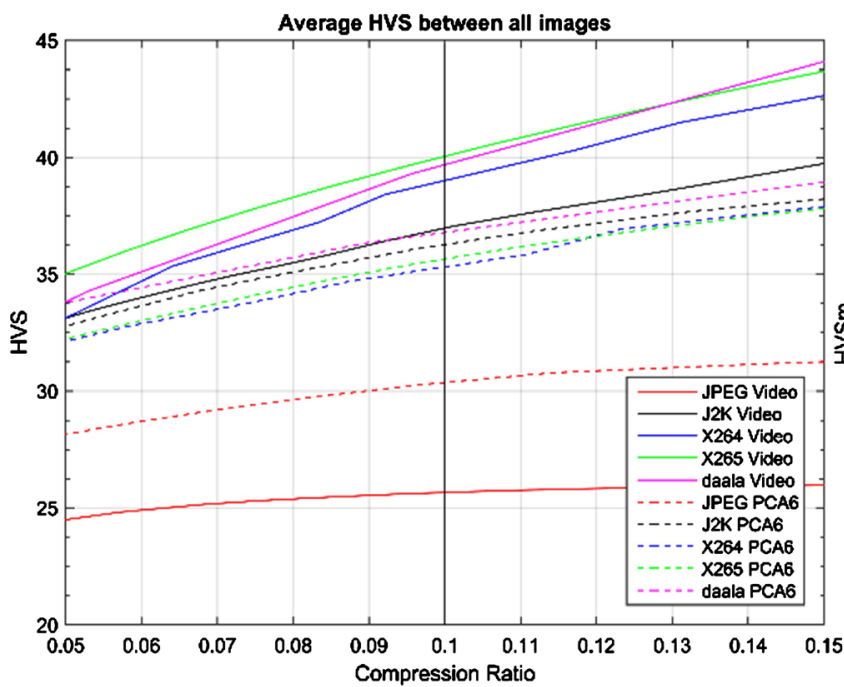

(c)

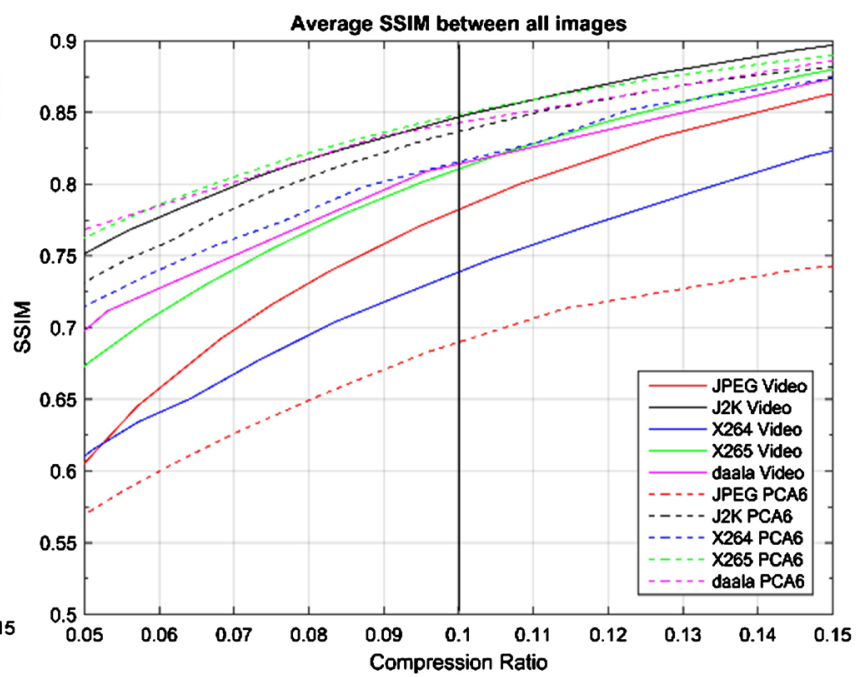

(b)

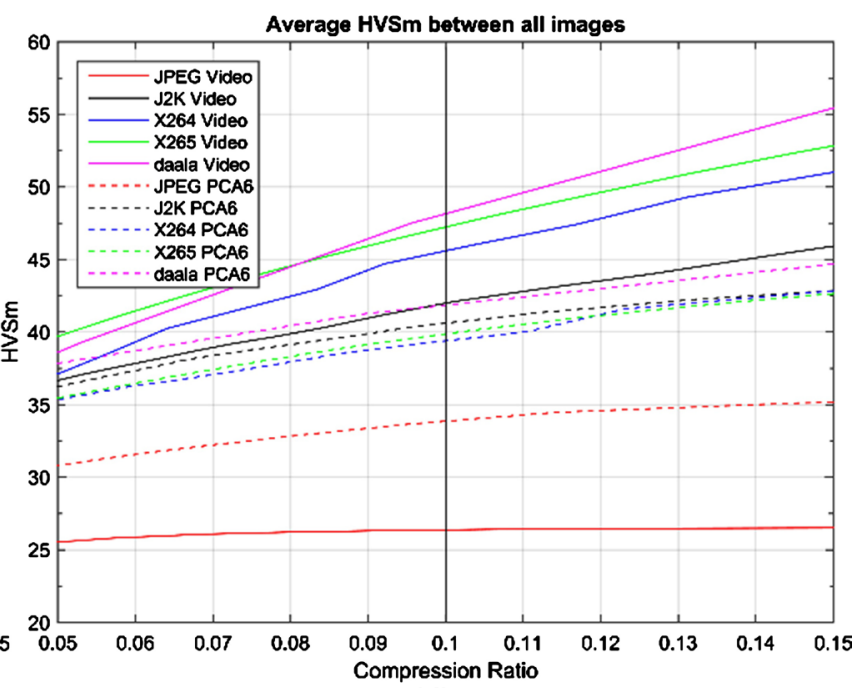

(d)

Figure 18. Comparison video and 2-step approaches for 20 left images near the 0.1 compression ratio region. (a) Averaged PSNR in $\mathrm{dB}$ of left images; (b) Averaged SSIM of left images; (c) Averaged HVS in dB of left images; (d) Averaged HVS-m in dB of left images. 


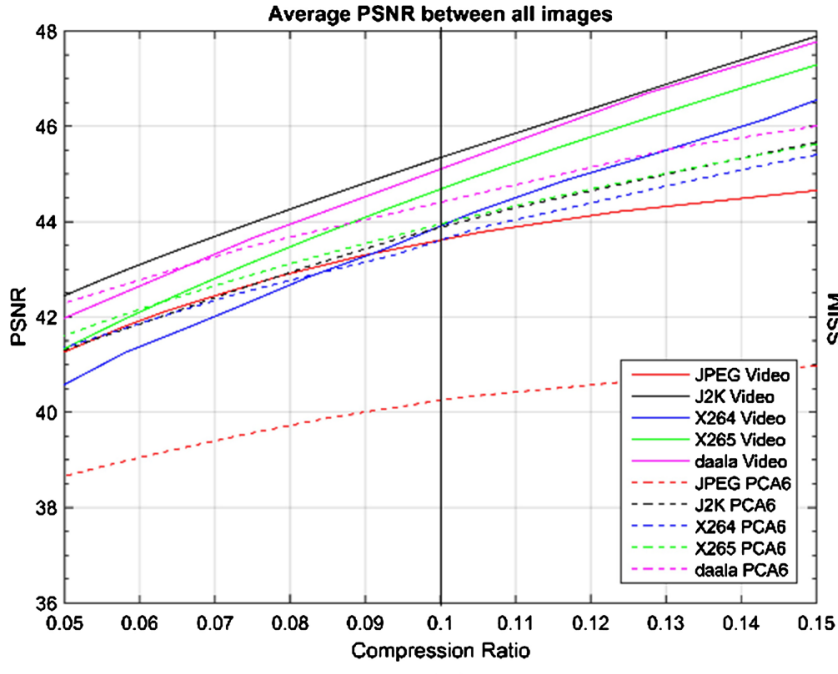

(a)

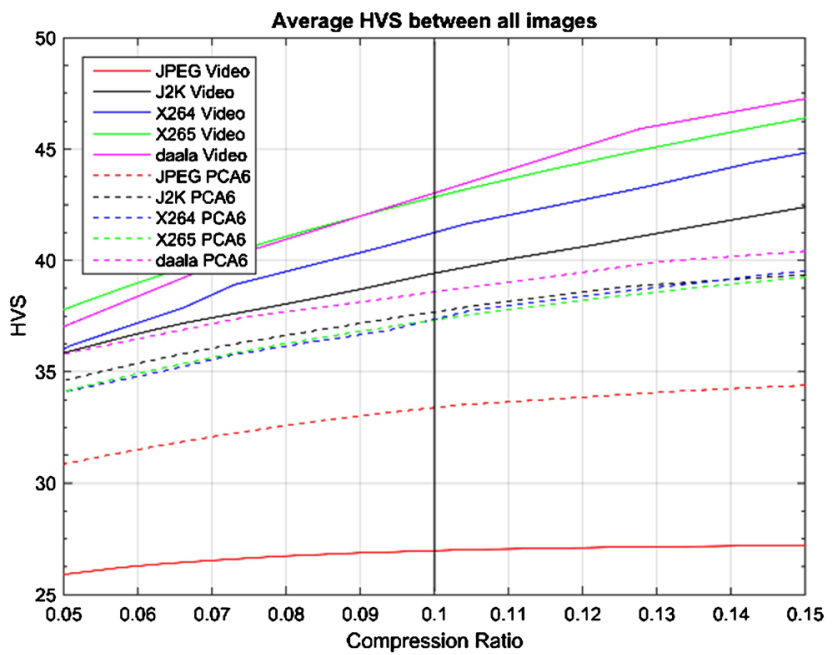

(c)

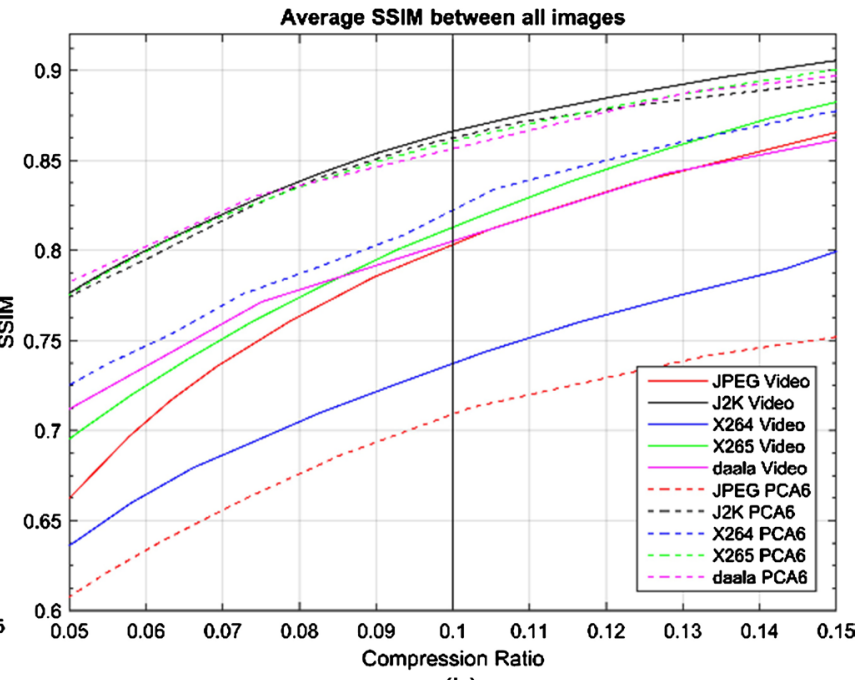

(b)

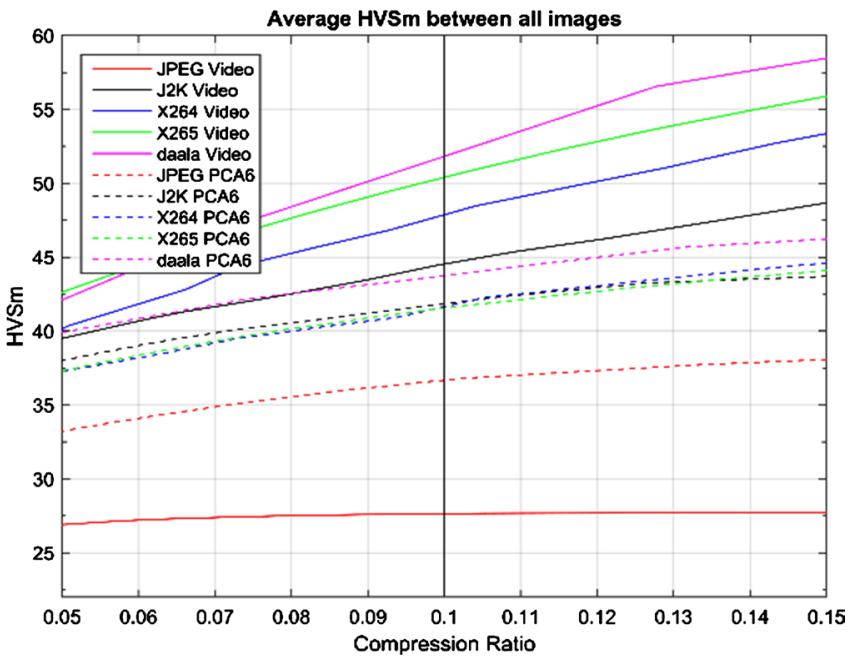

(d)

Figure 19. Comparison video and 2-step approaches for 20 right images near the 0.1 compression ratio region. (a) Averaged PSNR in $\mathrm{dB}$ of right images; (b) Averaged SSIM of right images; (c) Averaged HVS in $\mathrm{dB}$ of right images; (d) Averaged HVS-m in $\mathrm{dB}$ of right images.

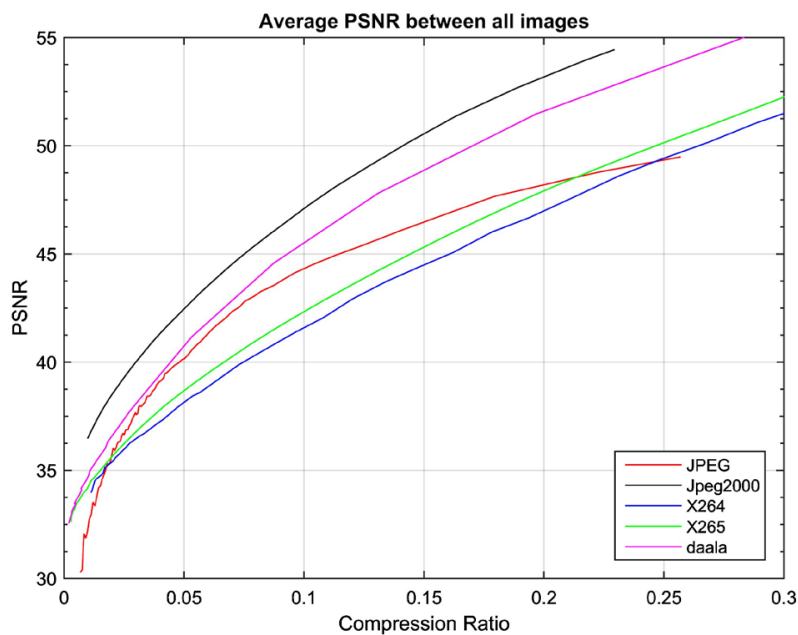

(a)

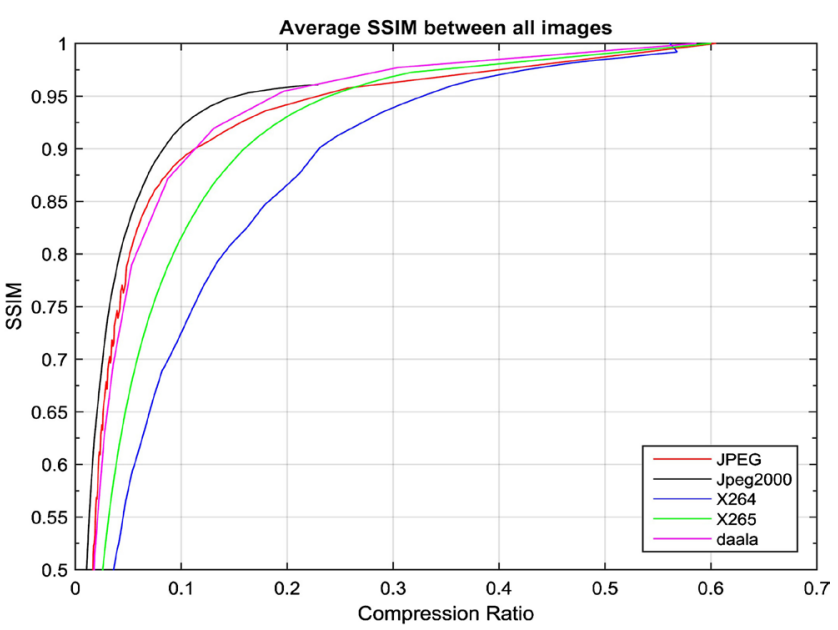

(b) 


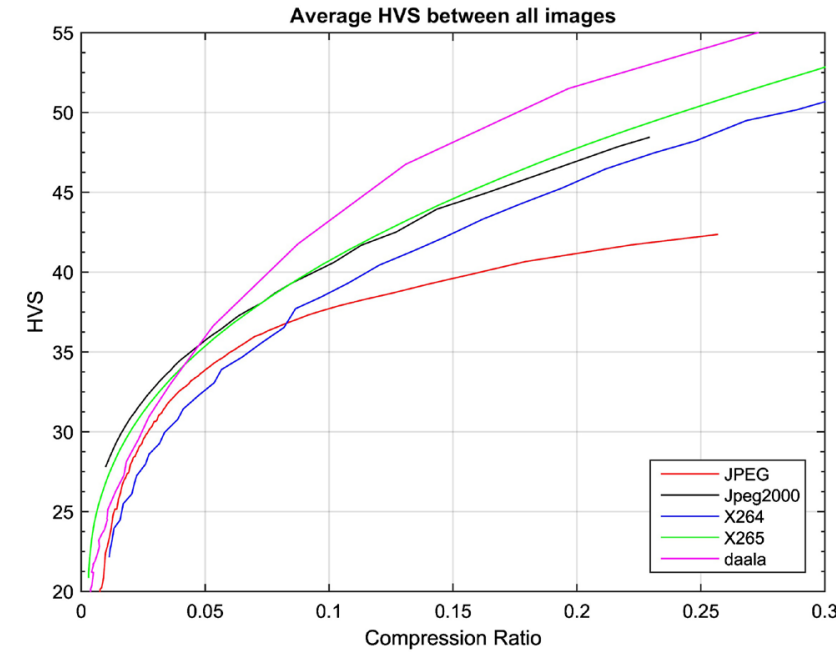

(c)

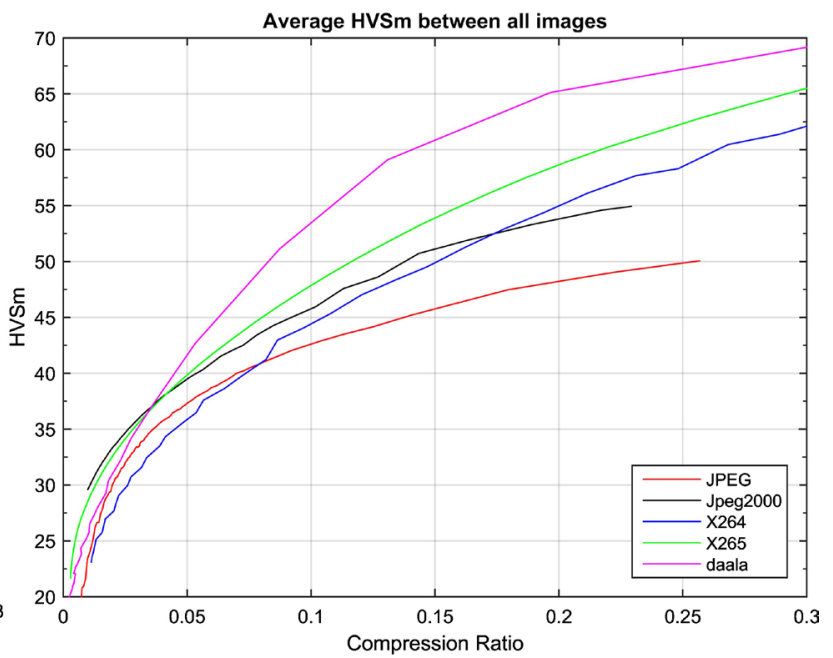

(d)

Figure 20. Comparison of different algorithms for 20 left RGB images. (a) Averaged PSNR in dB of left images; (b) Averaged SSIM of left images; (c) Averaged HVS in dB of left images; (d) Averaged HVS-m in dB of left images.

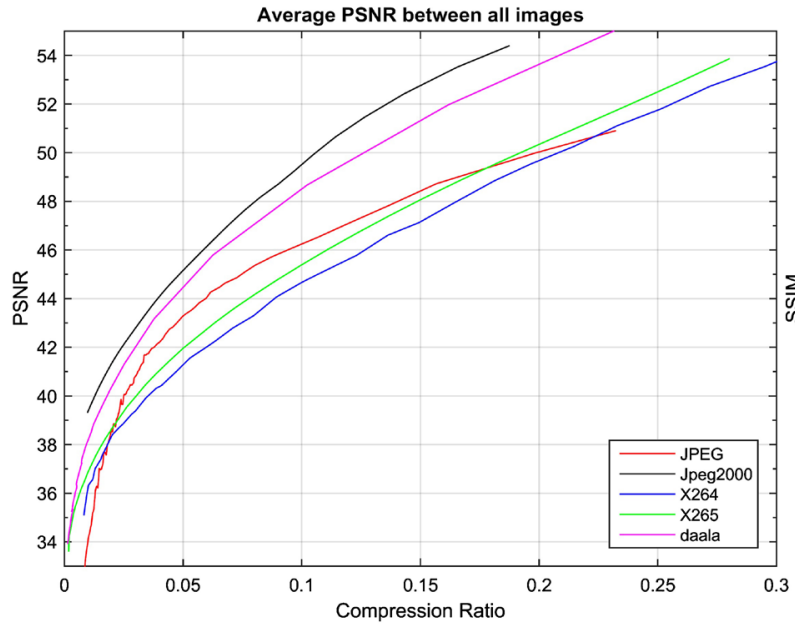

(a)

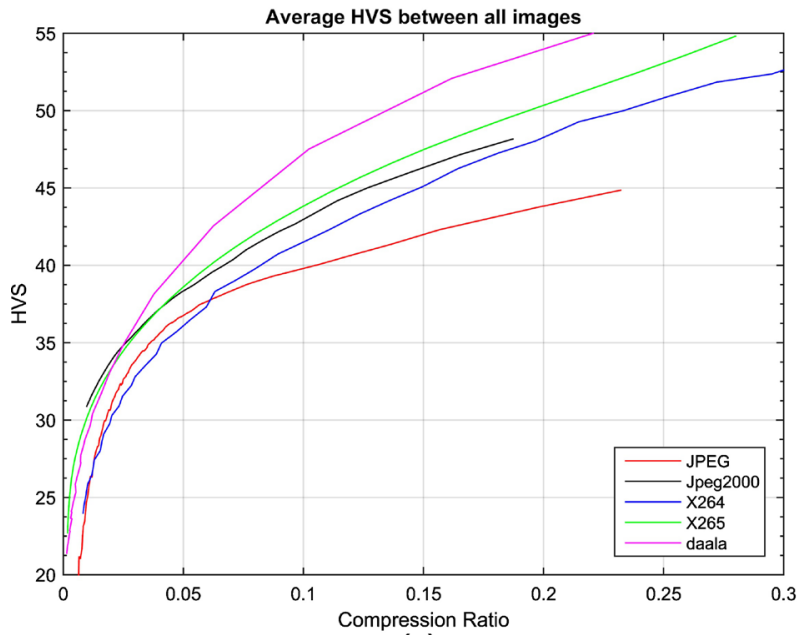

(c)

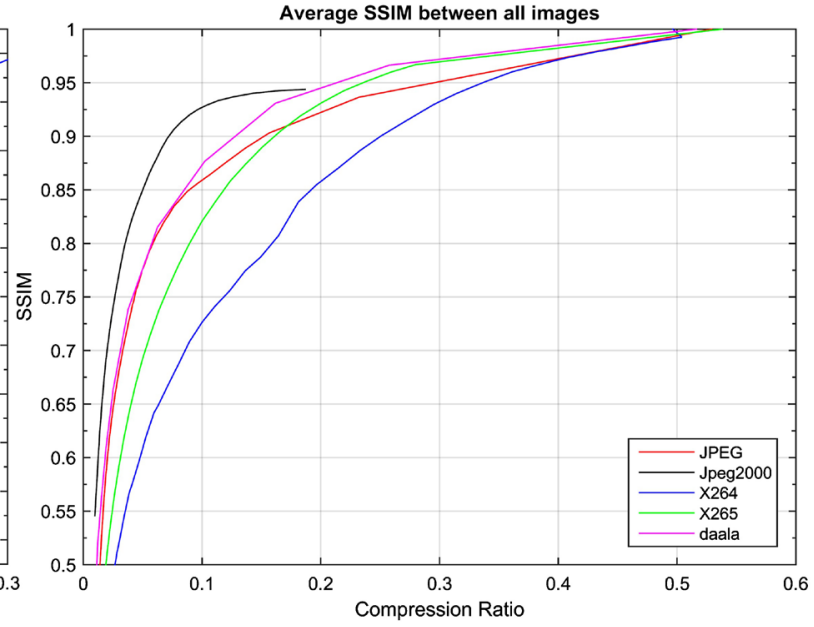

(b)

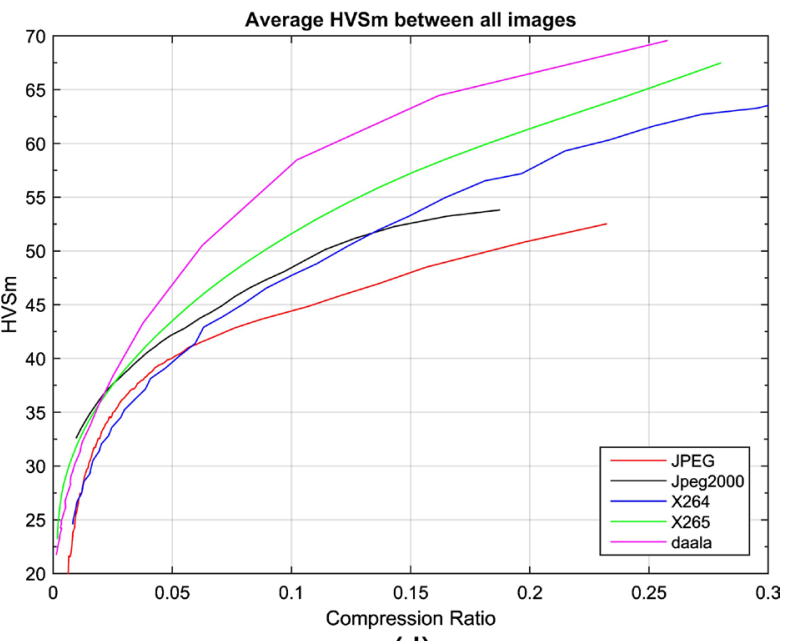

(d)

Figure 21. Comparison of different algorithms for 20 right RGB images. (a) Averaged PSNR in dB of right images; (b) Averaged SSIM of right images; (c) Averaged HVS in dB of right images; (d) Averaged HVS-m in dB of right images. 
Table 8. Performance metrics of five codecs at 0.1 compression ratio for left Mastcam RGB images. Bold numbers indicate the best performing method.

\begin{tabular}{ccccc}
\hline & PSNR (dB) & SSIM & HVS (dB) & HVSm (dB) \\
\hline JPEG & 44.37 & 0.89 & 38 & 42.5 \\
JPEG2000 & 47.1 & $\mathbf{0 . 9 3}$ & 40.5 & 45.62 \\
X264 & 41.56 & 0.73 & 38.75 & 44.37 \\
X265 & 42.4 & 0.82 & 40.75 & 48 \\
Daala & 45.63 & 0.88 & $\mathbf{4 3 . 1 3}$ & $\mathbf{5 3 . 2 5}$ \\
\hline
\end{tabular}

Table 9. Performance metrics of five codecs at 0.1 compression ratio for right Mastcam RGB images. Bold numbers indicate the best performing method.

\begin{tabular}{ccccc}
\hline & PSNR (dB) & SSIM & HVS (dB) & HVSm (dB) \\
\hline JPEG & 46.3 & 0.86 & 39.8 & 44.37 \\
JPEG2000 & 49.5 & $\mathbf{0 . 9 3}$ & 43 & 48.7 \\
X264 & 44.6 & 0.73 & 41.5 & 47.8 \\
X265 & 45.4 & 0.83 & 43.75 & 51.7 \\
Daala & 48.5 & 0.88 & $\mathbf{4 7 . 2}$ & $\mathbf{5 8 . 1 3}$ \\
\hline
\end{tabular}

ratio, J2K performed the best in terms of PSNR and SSIM. However, in terms of HVS and HVSm, Daala is the best performing one. It is also somewhat surprising to notice that JPEG is consistently the third best one in all metrics.

To subjectively evaluate the different codecs, we include three case studies. Case 1 is for compression ratio near 0.1 compression ratio. Figure 22 shows the original and 5 reconstructed images from JPEG, J2K, X264, X265, and Daala. We observe no perceptual loss of quality as compared to the original image. Case 2 is for compression ratio near 0.05 compression ratio. Again, it is still difficult to spot any artifacts between the reconstructed images and the original images shown in Figure 23. Case 3 is for compression ratio near 0.0251 compression ratio, which corresponds to 40 to 1 compression. In this case, we start to visualize some artifacts (Figure 24) in JPEG, X264, and X265. However, Daala and J2K still do not have visible artifacts.

\subsection{Discussions}

\section{- Comparison of different approaches}

In this paper, we propose a new video approach to compressing Mastcam multispectral images and compare with earlier approaches (PCA only, SB, and two-step). Through extensive experiments using actual Mastcam images, we observed that the SB approach yielded slightly better performance than others.

\section{- Comparison of different codecs}

In each approach, we have compared five codecs. Amongst them, we observed that Daala has the best performance in terms of HVS and HVSm. The improvement of Daala over JPEG is more than $5 \mathrm{dBs}$ in those metrics at 10 to 1 compression. 


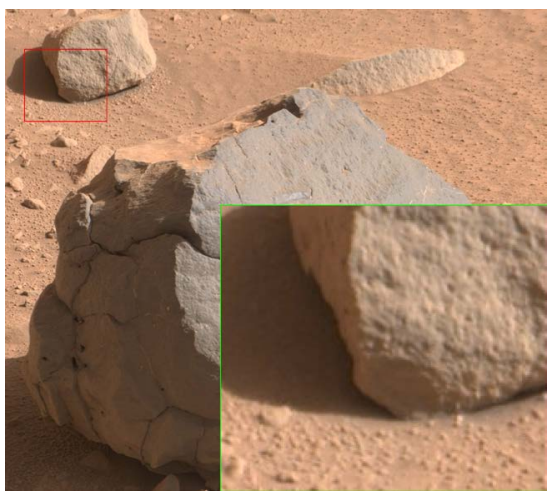

Original

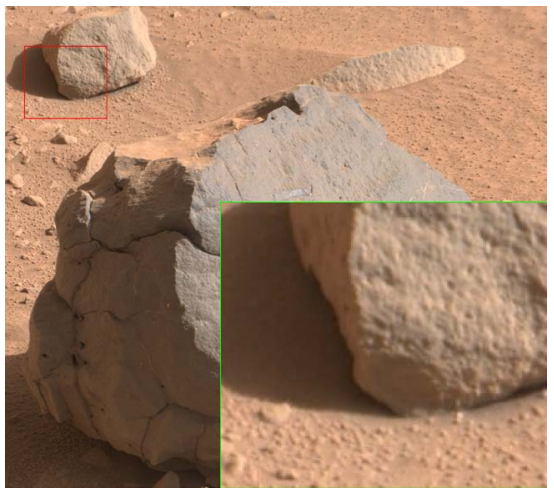

Reconstructed using J2K at CR of 0.099

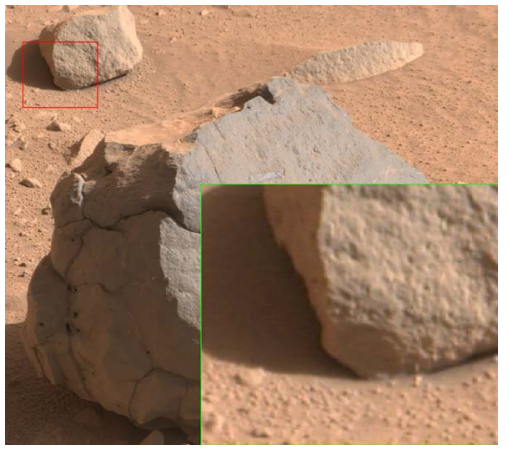

Reconstructed using X264 at CR of 0.105

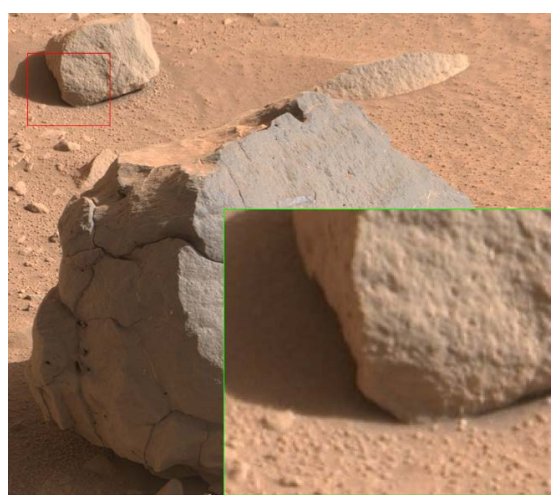

Reconstructed using JPEG at CR of 0.108

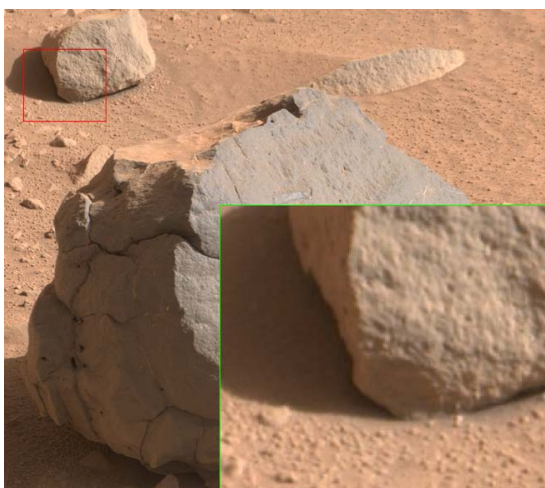

Reconstructed using Daala at CR of 0.103

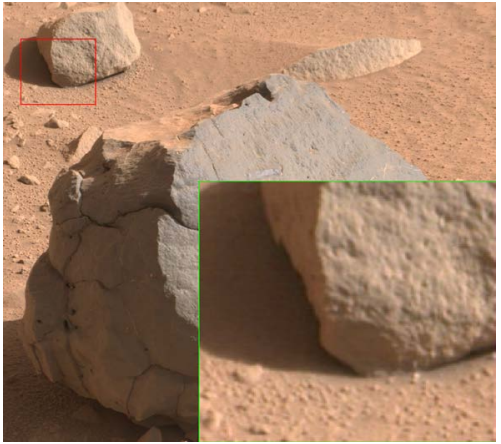

Reconstructed using X265 at CR of 0.0998

Figure 22. Visual comparison of different algorithms around a compression ratio of 0.1 (10 to 1 compression).

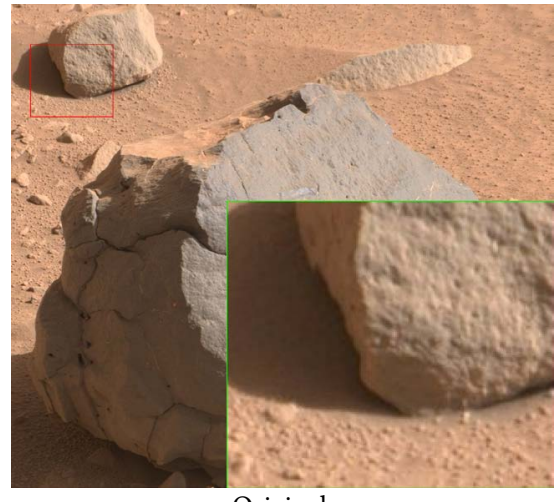

Original

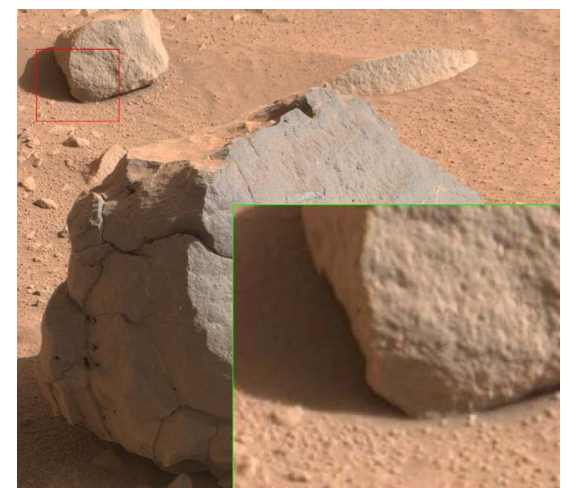

Reconstructed using JPEG at CR of 0.0497 


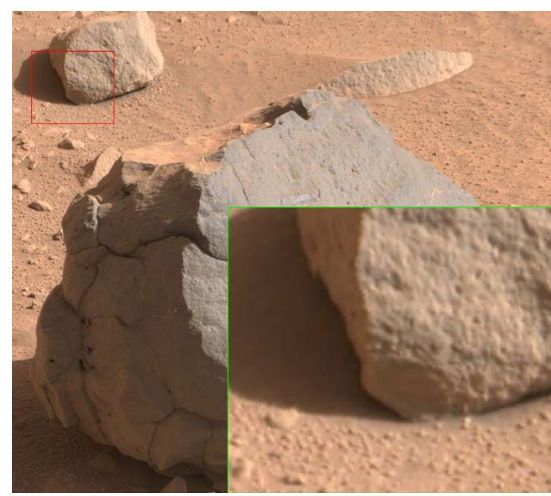

Reconstructed using J2K at CR of 0.0495

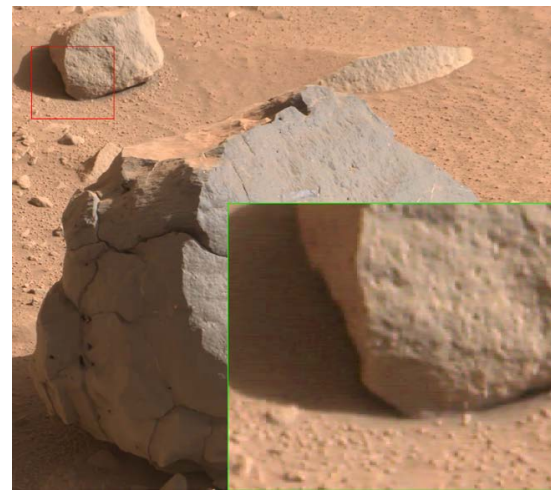

Reconstructed using X264 at CR of 0.0536

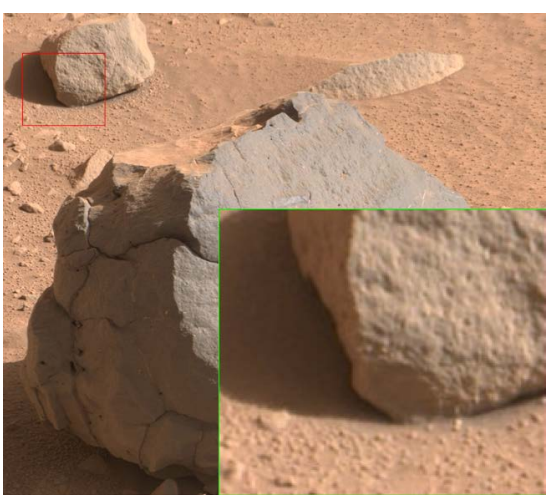

Reconstructed using Daala at CR of 0.0523

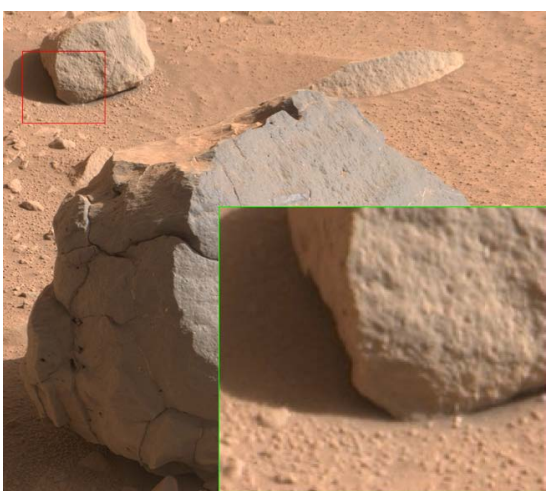

Reconstructed using X265 at CR of 0.0474

Figure 23. Visual comparison of different algorithms around a compression ratio of 0.05 (20 to 1 compression).

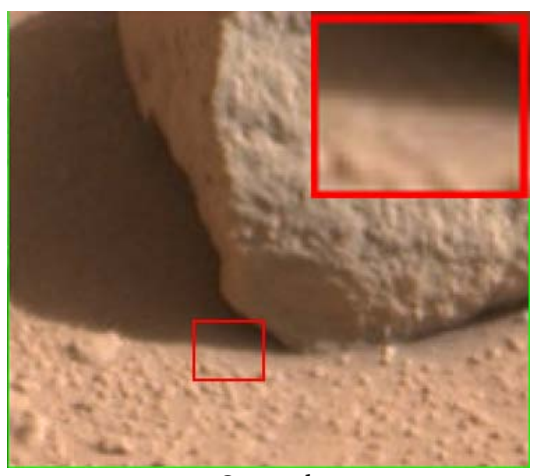

Original

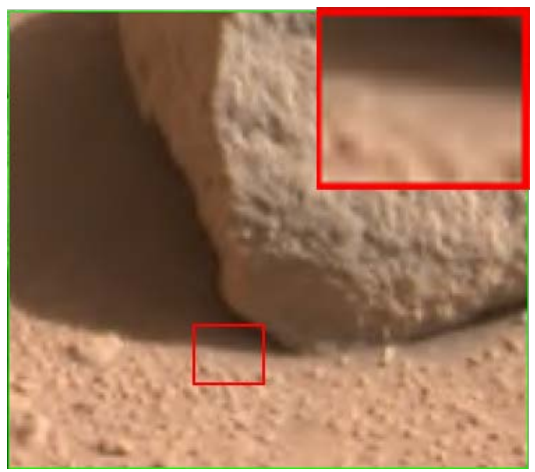

Reconstructed using J2K at CR of 0.0247

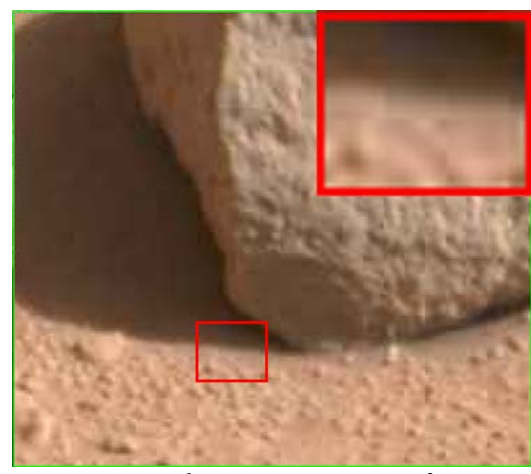

Reconstructed using JPEG at CR of 0.0247

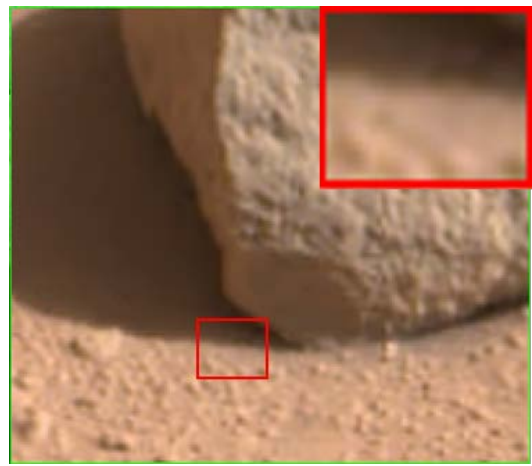

Reconstructed using Daala at CR of 0.0263 


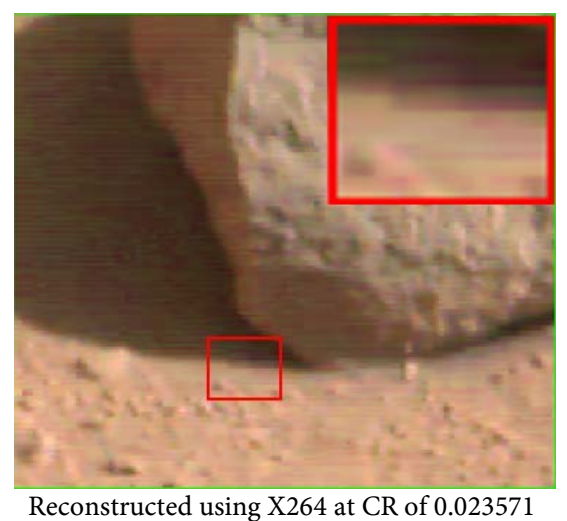

Reconstructed using X264 at CR of 0.023571

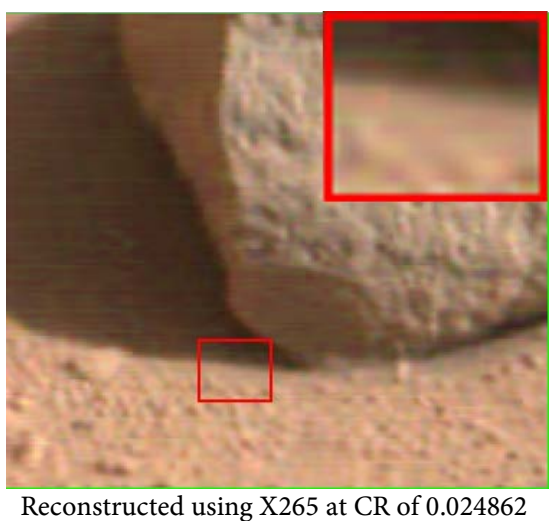

Reconstructed using X265 at CR of 0.024862

Figure 24. Visual comparison of different algorithms around a compression ratio of 0.025 (40 to 1 compression).

Since HVS and HVSm correlate well with human perception, we believe Daala is a good candidate to replace JPEG. For the two conventional metrics (PSNR and SSIM), we occasionally observed that JPEG and J2K has slight edge over other codecs. It is somewhat surprising to notice that the two popular codecs, X264 and X265, did not yield good results at 10 to 1 compression. However, they did sometimes have reasonable performance at lower compression cases such as 5 to 1 or 3 to 1 .

\section{- Computational Complexity}

Daala is DCT based and is hence amenable to parallel processing. J2K, on the other hand, is a wavelet based approach that requires the whole image. Hence, Daala is more efficient for fast processing.

\section{- Subjective Comparisons}

Through visual experiments using RGB images, we noticed that at 10:1 compression, all codecs have almost no loss. Even at 20:1 compression, it is still hard to notice any artifacts. However, at 40 to 1 compression, JPEG, X264 and X265 start to see some color distortions and block artifacts. Daala and J2K still performed reasonably well at 40:1.

\section{Conclusions}

One key objective in our research is to achieve perceptually lossless compression with 10:1 compression ratio for Mastcam multispectral images. We have evaluated four approaches (Video, SB, PCA only, and two-step). Five codecs (JPEG, J2K, X264, X265, Daala) using four performance metrics (PSNR, SSIM, HVS, HVSm). From our extensive experiments, it can be seen that SB approach with Daala performed the best, following by J2K. Subjective evaluations showed that perceptually lossless compression can be attached even at 20:1 compression. However, we recommend that 10:1 compression should be deployed because we believe the NASA wants to preserve the fidelity of the images as much as possible. At 10: 1 compression, it is 3 or 4 times better than lossless compression in terms of bandwidth saving. 
One future direction is to investigate how we can create a customized package for NASA. The package will essentially replace JPEG.

\section{Acknowledgements}

This research was supported by NASA Jet Propulsion Laboratory under contract \# 80NSSC17C0035. The views, opinions and/or findings expressed are those of the author(s) and should not be interpreted as representing the official views or policies of NASA or the US Government.

\section{Conflicts of Interest}

The authors declare no conflicts of interest regarding the publication of this paper.

\section{References}

[1] Strang, G. and Nguyen, T. (1997) Wavelets and Filter Banks. Wellesley-Cambridge Press, Wellesley, MA.

[2] Bell, J.F., et al. (2017) The Mars Science Laboratory Curiosity Rover Mast Camera (Mastcam) Instruments: Pre-Flight and in-Flight Calibration, Validation, and Data Archiving. AGU Journal Earth and Space Science, 4, 396-452. https://doi.org/10.1002/2016EA000219

[3] Ayhan, B., Kwan, C. and Vance, S. (2015) On the Use of a Linear Spectral Unmixing Technique for Concentration Estimation of APXS Spectrum. Journal of Multidisciplinary Engineering Science and Technology, 2, 2469-2474.

[4] Wang, W., Li, S., Qi, H., Ayhan, B., Kwan, C. and Vance, S. (2014) Revisiting the Preprocessing Procedures for Elemental Concentration Estimation Based on CHEMCAM LIBS on MARS Rover. 2014 6th Workshop on Hyperspectral Image and Signal Processing: Evolution in Remote Sensing (WHISPERS), Lausanne, Switzerland, 24-27 June 2014, 1-4. https://doi.org/10.1109/WHISPERS.2014.8077520

[5] Wang, W., Ayhan, B., Kwan, C., Qi, H. and Vance, S. (2014) A Novel and Effective Multivariate Method for Compositional Analysis Using Laser Induced Breakdown Spectroscopy. IOP Conference Series: Earth and Environmental Science, 17, Article ID: 012208. https://doi.org/10.1088/1755-1315/17/1/012208

[6] Ayhan, B., Dao, M., Kwan, C., Chen, H., Bell, J.F. and Kidd, R. (2017) A Novel Utilization of Image Registration Techniques to Process Mastcam Images in Mars Rover with Applications to Image Fusion, Pixel Clustering, and Anomaly Detection. IEEE Journal of Selected Topics in Applied Earth Observations and Remote Sensing, 10, 4553-4564. https://doi.org/10.1109/JSTARS.2017.2716923

[7] Kwan, C., Dao, M., Chou, B., Kwan, L.M. and Ayhan, B. (2017) Mastcam Image Enhancement Using Estimated Point Spread Functions. 2017 8th IEEE Annual Ubiquitous Computing, Electronics \& Mobile Communication Conference, New York City, 19-21 October 2017, 186-191.

https://doi.org/10.1109/UEMCON.2017.8249023

[8] Kwan, C., Chou, B. and Ayhan B. (2018) Enhancing Stereo Image Formation and Depth Map Estimation for Mastcam Images. 2018 9th IEEE Annual Ubiquitous Computing, Electronics \& Mobile Communication Conference, New York City, 8-10 November 2018, 566-572. https://doi.org/10.1109/UEMCON.2018.8796542

[9] Kwan, C. and Larkin, J. (2018) Perceptually Lossless Compression for Mastcam Im- 
ages. 2018 9th IEEE Annual Ubiquitous Computing, Electronics \& Mobile Communication Conference, New York City, 8-10 November 2018, 559-565. https://doi.org/10.1109/UEMCON.2018.8796824

[10] JPEG. http://en.wikipedia.org/wiki/JPEG

[11] JPEG-2000. http://en.wikipedia.org/wiki/JPEG_2000

[12] X264. http://www.videolan.org/developers/x264.html

[13] X265. https://www.videolan.org/developers/x265.html

[14] Tran, T.D., Liang, J. and Tu, C. (2003) Lapped Transform via Time-Domain Preand Post-Filtering. IEEE Transactions on Signal Processing, 51, 1557-1571.

https://doi.org/10.1109/TSP.2003.811222

[15] Daala. http://xiph.org/daala/

[16] Kwan, C., Larkin, J., Budavari, B. and Chou, B. (2019) Compression Algorithm Selection for Multispectral Mastcam Images. Signal \& Image Processing. An International Journal (SIPI), 10, 1-14. https://doi.org/10.5121/sipij.2019.10101

[17] Kwan, C. and Larkin, J. (2019) New Results in Perceptually Lossless Compression of Hyperspectral Images. Journal of Signal and Information Processing, 10, 96-124. https://doi.org/10.4236/jsip.2019.103007

[18] Ayhan, B., Kwan, C. and Zhou, J. (2018) A New Nonlinear Change Detection Approach Based on Band Ratioing. Algorithms and Technologies for Multispectral, Hyperspectral, and Ultraspectral Imagery XXIV, No. 1064410.

[19] Haykin, S. (1993) Neural Networks and Learning Machines. Pearson Education, Boston, MA.

[20] Wu, J., Liang, Q. and Kwan, C. (2012) A Novel and Comprehensive Compressive Sensing Based System for Data Compression. 2012 IEEE Globecom Workshops, Anaheim, CA, 3-7 December 2012, 1420-1425. https://doi.org/10.1109/GLOCOMW.2012.6477792

[21] Zhou, J. and Kwan, C. (2018) A Hybrid Approach for Wind Tunnel Data Compression. 2018 Data Compression Conference, Snowbird, UT, 27-30 March 2018, 435. https://doi.org/10.1109/DCC.2018.00088

[22] Kwan, C. and Luk Y. (2018) Hybrid Sensor Network Data Compression with Error Resiliency. 2018 Data Compression Conference, Snowbird, UT, 27-30 March 2018, 416. https://doi.org/10.1109/DCC.2018.00069

[23] Ponomarenko, N., Silvestri, F., Egiazarian, K., Carli, M., Astola, J. and Lukin, V. (2007) On between-Coefficient Contrast Masking of DCT Basis Functions. Proceedings of the Third International Workshop on Video Processing and Quality Metrics for Consumer Electronics VPQM-07, Scottsdale, AZ, 13-15 January 2010.

[24] JPEG-XR. http://en.wikipedia.org/wiki/JPEG_XR

[25] VP8. http://en.wikipedia.org/wiki/VP8

[26] VP9. http://en.wikipedia.org/wiki/VP9

[27] Kwan, C., Li, B., Xu, R., Tran, T. and Nguyen, T. (2001) Very Low-Bit-Rate Video Compression Using Wavelets. Wavelet Applications VIII, 4391, 76-180. https://doi.org/10.1117/12.421197

[28] Kwan, C., Li, B., Xu, R., Tran, T. and Nguyen, T. (2001) SAR Image Compression Using Wavelets. Wavelet Applications VIII, 4391, 349-357. https://doi.org/10.1117/12.421215

[29] Kwan, C., Li, B., Xu, R., Li, X., Tran, T. and Nguyen, T.Q. (2006) A Complete Image Compression Codec Based on Overlapped Block Transform. EUROSIP Journal of 
Applied Signal Processing, 2006, Article No. 010968. https://doi.org/10.1155/ASP/2006/10968

[30] Kwan, C., Shang, E. and Tran, T. (2018) Perceptually Lossless Image Compression with Error Recovery. 2nd International Conference on Vision, Image and Signal Processing, Las Vegas, NV, 27-29 August 2018, Article No. 16. https://doi.org/10.1145/3271553.3271602

[31] Kwan, C., Shang, E. and Tran, T. (2018) Perceptually Lossless Video Compression with Error Concealment. 2nd International Conference on Vision, Image and Signal Processing, Las Vegas, NV, 27-29 August 2018, Article No. 19. https://doi.org/10.1145/3271553.3271622 